\title{
A human skeletal muscle interactome centered on proteins involved in muscular dystrophies: LGMD interactome
}

Gaëlle Blandin 1,2, Sylvie Marchand ${ }^{1}$, Karine Charton ${ }^{1}$, Nathalie Danièle ${ }^{1}$, Evelyne Gicquel', Jean-Baptiste Boucheteil ${ }^{1}$, Azéddine Bentaib', Laetitia Barrault', Daniel Stockholm¹, Marc Bartoli ${ }^{1,2}$ and Isabelle Richard ${ }^{1 *}$

\begin{abstract}
Background: The complexity of the skeletal muscle and the identification of numerous human disease-causing mutations in its constitutive proteins make it an interesting tissue for proteomic studies aimed at understanding functional relationships of interacting proteins in both health and diseases.

Method: We undertook a large-scale study using two-hybrid screens and a human skeletal-muscle cDNA library to establish a proteome-scale map of protein-protein interactions centered on proteins involved in limb-girdle muscular dystrophies (LGMD). LGMD is a group of more than 20 different neuromuscular disorders that principally affect the proximal pelvic and shoulder girdle muscles.

Results and conclusion: The interaction network we unraveled incorporates 1018 proteins connected by 1492 direct binary interactions and includes 1420 novel protein-protein interactions. Computational, experimental and literature-based analyses were performed to assess the overall quality of this network. Interestingly, LGMD proteins were shown to be highly interconnected, in particular indirectly through sarcomeric proteins. In-depth mining of the LGMD-centered interactome identified new candidate genes for orphan LGMDs and other neuromuscular disorders. The data also suggest the existence of functional links between LGMD2B/dysferlin and gene regulation, between LGMD2C/ $/$-sarcoglycan and energy control and between LGMD2G/telethonin and maintenance of genome integrity. This dataset represents a valuable resource for future functional investigations.
\end{abstract}

Keywords: Interactome, Muscular dystrophies, Yeast-two hybrid

\section{Background}

The skeletal muscle tissue with its movement generation capacity is the organ of voluntary action but it plays also a major role in metabolic homeostasis. It is composed of long multinucleated cells, the myofibers, which possess a highly structured organization to ensure the dynamics and coordination of muscle contraction and resistance to resulting physical stresses. Notwithstanding its very well organized structure, the muscular tissue presents an important plasticity, which is necessary for adaptation to physical and metabolic constraints. These combined characteristics of structure and plasticity depend on the concerted action of protein complexes and of metabolic

\footnotetext{
* Correspondence: richard@genethon.fr

'Généthon CNRS UMR8587, 1, rue de l'Internationale, Evry 91000, France Full list of author information is available at the end of the article
}

and signaling pathways. These features together with the complexity of the skeletal muscle organization and its connection with human disorders makes it an interesting tissue for proteomic studies aimed at understanding functional relationships of interacting proteins in both health and diseases.

During the last decade, an increasing number of studies have been carried out to produce and analyze largescale protein-protein interaction networks in various bacterial and eukaryotic model systems. Several studies have investigated the human interacting-proteins network, either at a genome-wide scale [1-3] or with the aim of exploring a targeted interaction network [4-6]. Remarkably, several disease-targeted projects have proven to offer a very powerful strategy to address the role and function of the proteins involved, as exemplified

\section{Biomed Central}


by Huntington's disease [5] and in spinocerebellar ataxia [6]. These works have capitalized on several highthroughput technologies (yeast-two hybrid ( $\mathrm{Y} 2 \mathrm{H})$ system and Tandem Affinity Purification) to detect binary protein-protein interactions (PPI) or protein complexes, and have combined them with computational methods to propose "interactome" networks.

As an approach towards the identification of skeletal muscle functional networks, we undertook a large-scale study to establish a proteome-scale map of proteinprotein interactions centered on proteins involved in limb-girdle muscular dystrophies (LGMD). LGMD is a group of neuromuscular disorders with autosomal dominant (LGMD1) or recessive (LGMD2) inheritance modes that principally affect the proximal pelvic and shoulder girdle muscles (for a recent review see [7]). More than twenty different genetic entities were identified so far but it is estimated that $30-40 \%$ of patients clinically diagnosed with LGMD still do not have a genetic signature of their disease [8] with at least $25 \%$ of families who are not linked to any known locus and $40 \%$ of isolated cases with no detected mutation in any known LGMD gene. The known LGMD-causing genes encode proteins expressed in a variety of cellular compartments and involved in diverse biological functions that are not yet fully understood. Yet, the present knowledge has highlighted the importance of the components of the dystrophin-glycoprotein complex and $\alpha$-dystrophycan glycosyltransferases for membrane stability, and the implication of other LGMD proteins in processes such as regulation of sarcomere structure or nuclear stability, for the survival of the muscle fibers [9]. No curative treatment is currently available for LGMD, which pleads for the elucidation of the pathological mechanisms implicated in the diseases in order to propose innovative therapeutic approaches.

In this context, we selected a large-scale strategy to identify novel protein-protein interactions that could shed light on the biological processes at the origin of LGMD pathogenesis. We selected 13 LGMD-causing proteins and related proteins and ran high-throughput $\mathrm{Y} 2 \mathrm{H}$ assays to build a first interactome network that we further expanded by performing additional secondary and tertiary $\mathrm{Y} 2 \mathrm{H}$ screenings with new bait proteins of interest identified as preys in the primary screenings. The resulting LGMD-centered interaction network was established by combining results of 87 screenings based on 76 different bait proteins and incorporates 1018 proteins connected by 1492 direct binary interactions. Using both experimental and bioinformatics tools, we assessed the overall quality of this $\mathrm{Y} 2 \mathrm{H}$ network and isolated a high-confidence (HC) sub-network of 705 PPIs associating 497 proteins. The Y2H LGMD-centred network and its $\mathrm{HC}$ sub-network were compared to a literature-based network. Gene Ontology (GO) term analysis showed that the LGMD-centered interactome and especially its $\mathrm{HC}$ sub-network, is much more specifically enriched in proteins associated with the muscular tissue and the cytoskeleton than the literature-based dataset. Among the interesting outcomes of our study are the strong inter-connectivity of the LGMD proteins which, in addition to several direct links, present a number of indirect associations thanks to sarcomeric proteins, the identification of candidate genes for orphan neuromuscular disorders (NMDs) and the discovery of new possible functions for LGMD proteins. In particular, we suggest the existence of functional links between LGMD2B/dysferlin and gene regulation, between LGMD2C/ $\gamma$-sarcoglycan and energy control and between LGMD2G/telethonin and maintenance of genome integrity.

The protein interactions from this publication have been submitted to the IMEx (http://www.imexconsortium.org/) consortium through IntAct (pmid 22121220) and assigned the identifier IM-16425. The entire network can be browsed using the PIMRider software at http://pimr. hybrigenics.com.

\section{Methods}

\section{$\mathrm{Y} 2 \mathrm{H}$ bait design}

Bait design was organized in three successive rounds in which primary, secondary and tertiary baits were selected. Structural and functional domain predictions from TM-HMM [10], SignalP3.0 [11] or PFAM (PFAM 23.0, release 19/08/2008 [12]) were used to exclude hydrophobic trans-membrane domains, signal peptides and transcriptional trans-activation domains from bait constructs. In addition, to favor the identification of novel protein-protein interactions, we usually selected regions on the proteins that had not been previously documented for their functional role. For selection of secondary and tertiary bait proteins and for design of their bait sequences, we used additional bibliographic searches and other criteria computed from our $\mathrm{Y} 2 \mathrm{H}$ results such as the Predicted Biological Score (PBS) categories and information from Selected Interacting Domains (SID; see below section "Identification of interacting fragments and scoring of the interactions"). Some examples of bait candidates that came to our attention included targets of choice for therapeutic strategies such as proteins that participate in signaling pathways, proteins involved in various forms of myopathies or proteins expressed in typical muscle cellular compartments such as the sarcomere or sarcoplasmic reticulum.

\section{Bait cloning and library construction}

Bait sequences were PCR-amplified from MRC Gene Service or Invitrogen plasmids or from a random primed cDNA library obtained by reverse transcription of a poly 
(A) RNA library isolated from adult (Ambion AM7983) or 18-19 week-old fetal (Stratagene \#778020) human skeletal muscles. Bait PCR products were cloned in the pB27 plasmid, a plasmid derived from the original pBTM116 [13], as a LexA C-terminal fusion [14]. Plasmid DNA was purified with the QIAprep Spin Miniprep (QIAGEN), verified by full insert sequencing and introduced into the L40 $\mathrm{Gal} 4(M A T a)$ bait yeast strain [15]. Alternatively, prey fragments were directly extracted from the prey plasmid and subsequently cloned in pB27 to use them as secondary or tertiary baits.

The prey library in yeast was constructed from adult (Ambion AM7983) and fetal (Stratagene \#778020) human skeletal muscle poly(A) RNA. Random-primed cDNA fragments were prepared from these two RNA pools and cloned in the pP6 plasmid derived from the original pACT2 (Clontech) as a C-terminal fusion of the Gal4 transcription activating domain. Altogether, 90\% of the plasmids contained a cDNA insert with an average size of 600 bp. After amplification in Escherichia coli (50-100 million independent clones), the Y187 (MATalpha) yeast strain was transformed with an equimolar pool of the adult and fetal cDNA libraries. Ten million independent yeast colonies were then collected, pooled and stored at $-80^{\circ} \mathrm{C}$ as equivalent aliquot fractions of the same prey library. Validation of the prey library was performed by recapitulating several published interactions as described in [14]. Bait proteins belonging to different functional classes were used: a GTPase (Rac1), a transcription factor (TP53), a splicing factor (SF1) and a component of a E3-ligase complex (BTRC).

\section{Screening procedure and identification of prey fragments} $\mathrm{Y} 2 \mathrm{H}$ screens were performed using a mating method as described in [14] at the Hybrigenics facility (Hybrigenics, Paris). As first step, small-scale screenings were performed to assess toxicity and auto-activation capacity of the baits and to adjust selective pressure of the screens accordingly. In particular, the optimal concentration of 3-aminotriazol (3-AT) was determined prior to performing each large-scale screen. Auto-activating baits able to activate transcription of the reporter gene by themselves were identified and were not considered for large-scale screenings. Subsequently, each bait clone was tested in a full-size screen against an average of 103 million yeast prey clones, equivalent to ten-fold coverage of the library. All positive clones were picked and the corresponding prey fragments were PCR-amplified and sequenced at their $5^{\prime}$ and $3^{\prime}$ junctions. Sequence contigs were built and identified by comparison to the NCBI Human RefSeq database as described in [14].

\section{Identification of interacting fragments and scoring of the interactions}

Following contig assembly of positive clones, the common sequence shared by the assembled prey fragments was used to define the SID along each prey protein. Furthermore, for each interaction, a PBS was computed with E-values ranging from 0 to 1 to establish six distinct categories: PBS-A to -E (see [14] for details on calculation). The technically most reliable interactions were associated with the PBS-A, $-\mathrm{B}$ or $-\mathrm{C}$ categories (with $\mathrm{P}$ values $<1^{e-10}$ for PBS-A; $<1^{e-5}$ for PBS-B and $<1^{e-2.5}$ for PBS-C) and are found in two reciprocal and independent screens $(X->Y$ and $Y->X)$ and/or in interaction cycles $(X-Y$, $\mathrm{Y}-\mathrm{Z}$ and $\mathrm{X}-\mathrm{Z}$ ) and/or in a single screen but with many overlapping prey fragments. Interactions were assigned to the PBS-D category when they were supported either by a single experimental clone from a screen or by several clones bearing the same start and stop positions, the SID being identified by a singleton fragment instead of a family of several overlapping fragments. This PBS-D category corresponds to a heterogeneous group of interactions that theoretically could consist of technical false-positive interactions as well as true-positive interactions hardly detectable by $\mathrm{Y} 2 \mathrm{H}$ systems (due to constraints in tridimensional conformation of bait or prey domains, toxicity in yeast, poor mRNA representation of the prey in the library, ...). All the PBS-D should therefore be considered as putative unless validated by a second technique. The PBS-E category characterizes SID that have been found as prey in more than ten independent screens with unrelated bait proteins in all screenings performed with human libraries at the Hybrigenics facility. These interactions potentially represent possible false-positives of the $\mathrm{Y} 2 \mathrm{H}$ system as well as interactions with proteins known to be highly connected due to their biological function or with proteins containing a biochemically promiscuous motif. Finally, interactions with proteins or domains corresponding to known false positives of the $\mathrm{Y} 2 \mathrm{H}$ system as it is described above were removed from the data and from our analyses. Examples of yeast growth assays describing interactions with the different PBS categories using the same experimental procedures can be found in [16-18].

\section{Antibodies}

The antibodies used for immunoprecipitation of the baits are BD Biosciences anti-TCAP (T26820-050), Novocastra Laboratories Ltd anti-DYSF (NCL-Hamlet) and Santa Cruz Biotechnology anti-ABI1 (sc-30038), anti-ACTN2 (sc-15335), anti-DES (sc-14026), anti-MYOM1 (sc-30390) and anti-TCAP (sc-8725).

The antibodies used for prey detection by western blot are Abcam anti-SNAPIN (ab37496), Abnova anti-ADPGK 
(H00083440-M01), anti-APPL1 (H00026060-A01) and anti-ENO1 (H00002023-M04), Aviva anti-KBTBD10 (ARP38732_T100) and Santa Cruz Biotechnology antiKIF1B (sc28540) and anti-KTN1 (sc33562).

The antibodies used for immunochemistry and Duolink assays and their corresponding dilutions are: Abcam anti-CMYA5 (ab75351, 1:50) and anti-OPTN (ab23666, 1:100), Abgent anti-DGKD (AP8126b, 1:50), Abnova anti-DNAJB6 (H00010049-M01, 1:100) and anti-EEF1G (H00001937-M01, 1:50), Novocastra Laboratories Ltd anti-DYSF (NCL-Hamlet, 1:20), Proteintech Group anti-SNAPIN (10055-1 AP, 1:50), Santa Cruz Biotechnology anti-ACTN2 (sc-15335, 1:100), anti-ALMS1 (sc-54507, 1:50), anti-APPL1 (sc-67402, 1:50), anti-DES (sc-14026, 1:100), anti-FLNC (sc-48495, 1:100), antiKIF1B (sc-28540, 1:50), anti-MYOM1 (sc-30390, 1:100), anti-MYOM2 (sc-50435, 1:200) and anti-NEB (sc-28286, 1:100) and Sigma anti-NPHP3 (HPA009150, 1:75).

\section{Co-immunoprecipitation}

The bait proteins were isolated from R9 cell extracts (a gift from Dr. Anne Galy, Inserm U790, Evry, France) at myoblast or myotube stage (7 to 10 days of differentiation) or from gastrocnemius muscle excised from four week-old mice and homogenized in $6 \mathrm{ml}$ lysis buffer (Tris $20 \mathrm{mM}$, pH 7.5, NaCl $50 \mathrm{mM}$, EGTA $2 \mathrm{mM}$, Triton 1\%, Protease Inhibitor Cocktail (Complete mini, Roche), E64 $2 \mu \mathrm{M}$ ) using a FastPrep-24 apparatus (MP Biomedicals). The mouse samples correspond to a protocol approved by Genethon's ethics committee under the number CE11_014 and performed in accordance with the directive of 24 November 1986 (86/609/EEC) of the Council of the European Communities. After centrifugation of the lysates, $500 \mu \mathrm{g}$ to $1 \mathrm{mg}$ of proteins in $1 \mathrm{ml}$ were incubated with $30 \mu \mathrm{l}$ of protein $\mathrm{G}-$ Sepharose beads (Amersham) for $1 \mathrm{~h}$ to clear from nonspecific binders. The protein extract was then subjected to immunoprecipitation by $1 \mathrm{~h}$ incubation at $+4^{\circ} \mathrm{C}$ with 2 to $4 \mu \mathrm{g}$ of primary antibodies corresponding to the baits, then $30 \mu \mathrm{l}$ of protein $\mathrm{G}$ Sepharose beads (Amersham) were added and incubation was carried out for $2 \mathrm{~h}$ or overnight at $+4^{\circ} \mathrm{C}$.

After centrifugation at $1000 \mathrm{~g}$ for $5 \mathrm{~min}$, the immunocomplex was washed three times with $1 \mathrm{ml}$ of buffer and resuspended in $15 \mu \mathrm{l} 4 \mathrm{x}$ NuPAGE LDS sample buffer (Invitrogen) and dithiothreitol reducing agent. Samples were then heated at $+70^{\circ} \mathrm{C}$ for $10 \mathrm{~min}$ and centrifuged briefly. Protein complexes were separated by electrophoresis on SDS-PAGE NUPAGE 4-12\% Bis-Tris gel (Invitrogen). Transfer of the proteins was performed on PVDF membrane and verified by staining with Ponceau red. Immunostainings were performed with primary antibodies corresponding to prey and IRD-680 or 800 donkey anti-mouse, -rabbit or -goat as secondary antibodies according to LI-COR's protocol. Bands were then visualized with the Odyssey infra-red imaging system (LI-COR-Biosciences) at $700 \mathrm{~nm}$ (red) and $800 \mathrm{~nm}$ (green).

\section{Immunohistochemistry}

Indirect immunofluorescence microscopy assays were carried out on transversal cryosections prepared from normal human paravertebral striated muscles of a 13-year old female biopsy obtained from the biobank Myobank under the validation number AC-2008-87 from the French ministry of research (Institute of Myology, Paris). The sample was treated anonymously. Frozen slides were airdried for $30 \mathrm{~min}$ at room temperature, fixed with $4 \%$ PAF for $5 \mathrm{~min}$, washed $3 \times 5 \mathrm{~min}$ in PBS, incubated in a blocking buffer (4\% BSA, $0.02 \%$ Triton) for $30 \mathrm{~min}$, washed in PBS, then incubated with a biotin blocking solution (Vector Laboratories, SP-2001) for $15 \mathrm{~min}$ and washed in PBS for $5 \mathrm{~min}$. Slides were stained at room temperature for $1 \mathrm{~h}$ or at $+4^{\circ} \mathrm{C}$ overnight with primary antibodies diluted in the labeling solution (1\% BSA / PBS). Slides were then incubated with a donkey anti-mouse -Alexa 488 for dysferlin and a donkey (anti-rabbit or antigoat) biotinylated secondary antibody for its partner (dilution 1:1000) for $45 \mathrm{~min}$, washed 3x $5 \mathrm{~min}$ in PBS and stained with streptavidin coupled to Alexa-594 (Molecular Probes, dilution 1:500 in PBS) for $30 \mathrm{~min}$. For nucleic acid staining, slides were then incubated with TOPRO-3 (Molecular Probes, dilution 1:2000) for 5 min, washed $2 x$ $5 \mathrm{~min}$ in PBS and $1 \mathrm{x}$ in water for $2 \mathrm{~min}$. Slides were subsequently mounted in Fluoromount- $\mathrm{G}^{\mathrm{TM}}$ (SouthernBiotech, 0100-01). Images were acquired using the $40 x$ or $63 x$ objective of a Zeiss Axiovert 100 M. LSM.510 Meta laser scanning confocal microscope and the constructer software. Colocalization analyses were performed by statistical analysis of the correlation between the intensity values of red and green pixels in a dual- channel image. The JACop plug-in [19] for ImageJ (Rasband, W.S., ImageJ, U. S. National Institutes of Health, Bethesda, Maryland, USA, http://imagej.nih.gov/ij/, 1997-2011 ) was used to calculate Pearson's Correlation coefficient. Co-localization was defined as strong for $0.5<R \leq 1$, medium for $0.25<R \leq 0.5$ and low for $\mathrm{R} \leq 0.25$.

\section{Proximity ligation assays}

The Duolink ${ }^{\circledR}$ kit (Olink Bioscience) is based on the use of two unique and bi-functional probes called PLA ${ }^{\mathrm{TM}}$, each probe consisting of a secondary antibody attached to a unique synthetic oligonucleotide that acts as a reporter. After a 10 min fixation with paraformaldehyde $4 \%$ and blocking (BSA 5\% in PBS) steps, muscle sections were stained with one or two primary antibodies depending on the experiment (single protein detection or detection of interacting proteins) over-night at $+4^{\circ} \mathrm{C}$. 
After washing, the sections were incubated with the secondary oligonucleotide-linked antibodies (PLA probes) provided in the kit. The oligonucleotides bound to the antibodies were hybridized, ligated, amplified, and detected using a fluorescent probe (Detection Kit 563). Dots were detected with the Zeiss laser scanning confocal microscope and intensity signal counted using ImageJ software (http://imagej.nih.gov/ij/). A series of controls were performed for each analysis (bait antibody only, prey antibody only and negative control for which the primary antibody is omitted).

For quantification analysis: three images were acquired under the same conditions (laser power, PMT gain and pinhole) for each experiment. For each image, five fibers were randomly selected and used to count all positive spots within each compartment (total of 15 cells). The regions of interest (ROI) for membrane and cytoplasm compartments were separately delimitated manually and signal quantification was performed on all identified spots using the ImageJ software. For each compartment, we considered that the PPI was validated by the assay when the mean signal ratio between the PLA images of the PPI, "PPI signal", and the control images of the prey, "PREY signal", was superior to 0.2 , indicating that the interaction with the prey potentially recruited more than $20 \%$ of the interacting partner in the delimited compartment.

\section{Bioinformatics and statistical analyses}

IpScan [20] with Interpro 17.0 [21] was used to annotate the protein sequences. The SID coordinates were compared with the position of the different Interpro domains. Cytoscape tools (www.cytoscape.org) were used to infer connectivity, a parameter that indicates the number of proteins that directly interacts with a given protein. Comparison of PPIs identified by our $\mathrm{Y} 2 \mathrm{H}$ screenings with previously published PPIs was performed using the iRefWeb interface ([22]; http://wodaklab.org/iRefWeb/) by considering direct interaction found in mammals.

GO mapping and clustering were performed with the DAVID 6.7 web interface [23,24] using the Functional Annotation Clustering tool and the GOTERM_FAT annotation categories in order to filter the broadest GO terms. For the LGMD-centered dataset, a list of official gene symbols was used to identify the proteins and within each identified GO cluster, GO terms were analyzed in terms of hierarchy to identify the most specialized children terms common to all proteins within the cluster and these terms were reported as "shared GO" annotations (Additional file 1: Table S1). To analyze whether our datasets were statistically different from a random dataset, GO clustering was also performed with a list of Uniprot accessions for the 19220 human protein-coding genes (HGNC, www.genenames.org/). The number (Shared-i) of human proteins with which a given bait protein (Bait-I) shared a GO cluster was calculated for all three GO classes (BP, MF and CC) and all baits and the number of protein pairs not sharing a GO cluster was deduced (NonShared-i $=19220-\mathrm{Pi})$. The overall frequency of expected shared and non shared protein pairs was calculated as the ratio between the sum of Shared-i and the sum of all pairs $(76 \times 19220)$, and the sum of NonShared-i and the sum of all pairs, respectively. A Chi2 test $(\mathrm{P}<0.05)$ was used to compare expected values with observed values from the LGMD-centred dataset or the subset consisting of all PBS-A to -C categories.

GO enrichment analyses were performed using the DAVID Functional Annotation tool with Uniprot accession numbers as identifiers, the Homo sapiens background and the GOTERM_FAT annotation categories. Enrichment at $1 \%$ significance level was defined with a modified Fisher exact $P$ value (the "EASE" score) as recommended by the DAVID interface.

Statistical analysis of obtained proportions for the other analyses was done using the Fisher test function in R.

\section{Results}

\section{Bait design and screening procedure}

The procedure for choosing the bait protein sequences to be used as baits for our $\mathrm{Y} 2 \mathrm{H}$ screenings lied in three successive steps in which primary, secondary and tertiary baits were selected to perform three rounds of $\mathrm{Y} 2 \mathrm{H}$ screenings. First, we selected nine proteins involved in recessive LGMD forms and four proteins that were either known as LGMD-binding proteins or described as having a role in muscular atrophic processes (Table 1). Design of the baits excluded hydrophobic transmembrane domains, signal peptides and transcriptional trans-activation domains to ensure the best $\mathrm{Y} 2 \mathrm{H}$ screening conditions. We chose either full-length coding sequences or specific domains as bait, especially, for large proteins such as titin (TTN) and dysferlin (DYSF). Overall, we selected 20 primary bait domains.

For each bait domain, we first assessed its toxicity and auto-activation capacity by a small-scale $\mathrm{Y} 2 \mathrm{H}$ screen and then performed a large-scale $\mathrm{Y} 2 \mathrm{H}$ assay by screening a high-complexity cDNA prey library obtained by random priming of poly(A)+ RNA from adult and fetal human skeletal muscles that we constructed for this purpose. The bait interaction was tested against an average of 103 million prey clones to insure a ten-fold coverage of the prey library. Positive prey clones were sequenced and compared to the NCBI human RefSeq database for prey identification. Contig assembly of positive clones was performed to isolate the minimum interacting domain(s) on each prey sequence [Selected Interacting Domains (SID)]. We used clone coverage and local topology information to compute a confidence score [Predicted 
Table 1 A/Description of primary baits

\begin{tabular}{|c|c|c|c|}
\hline Protein name & $\begin{array}{l}\text { Protein symbol and } \\
\text { LGMD form }\end{array}$ & $\begin{array}{l}\text { Bait domain } \\
\text { coordinates }(\mathrm{aa})^{*}\end{array}$ & Bait domain description \\
\hline \multirow[t]{2}{*}{ calpain 3} & CAPN3 (LGMD2A) & T417-S643 & C2-like domain + exons15-16 \\
\hline & & M1-A822 & $\begin{array}{l}\text { full length protein with the C129S mutation in the autocatalytic } \\
\text { site }\end{array}$ \\
\hline \multirow[t]{3}{*}{ dysferlin } & DYSF (LGMD2B) & L2-1485 & $\mathrm{N}$-terminal DYSF regions containing the first three $\mathrm{C} 2$ domains \\
\hline & & Q851-D1200 & central DYSF domain \\
\hline & & I1145-L2026 & $\begin{array}{l}\text { C-terminal DYSF domain containing the last four C2 domains and } \\
\text { excluding the transmembrane span }\end{array}$ \\
\hline$\gamma$ - sarcoglycan & SGCG (LGMD2C) & M1-L36 & cytoplasmic domain \\
\hline a-sarcoglycan & SGCA (LGMD2D) & M313-H388 & cytoplasmic domain \\
\hline$\beta$-sarcoglycan & SGCB (LGMD2E) & E10-A65 & cytoplasmic domain \\
\hline$\delta$-sarcoglycan & SGCD (LGMD2F) & E1-Y36 & cytoplasmic domain \\
\hline titin-cap (telethonin) & TCAP (LGMD2G) & M1-G167 & full length protein \\
\hline tripartite motif-containing 32 & TRIM32 (LGMD2H) & L66-P654 & full length protein minus the RING domain \\
\hline \multirow[t]{5}{*}{ titin } & TTN (LGMD2J) & V97-K469 & exons 4-8 from the Z-Disc region \\
\hline & & 1741-G948 & exons $14-17$ from the Z-Disc region \\
\hline & & R2120-L2564 & exons 28-33 from the Z-Disc region \\
\hline & & A8831-E9158 & exons 108-114 from the N2A-PEVK region \\
\hline & & T32840-133423 & exons 358-363 from the Mline region \\
\hline ankyrin repeat domain 1 & ANKRD1 & M1-F319 & full length protein \\
\hline ezrin & $\mathrm{EZR}$ & M1-S536 & full length protein minus the actin-binding C-terminal domain \\
\hline F-box protein 32 (MAFbx) & $\mathrm{FBXO32}$ & M1-F356 & full length protein \\
\hline tripartite motif-containing 63 (MuRF1) & TRIM63 & M1-Q354 & full length protein \\
\hline
\end{tabular}

* amino acid coordinates refer to the translated products of the nucleotide sequences indicated in Additional file 2: Table S2.

Biological Score (PBS)] and classify each PPI into five categories: PBS-A, $-\mathrm{B}$ or $-\mathrm{C}$ for the most reliable interactions, PBS-D for putative interactions involving a single bait clone and PBS-E for interactions involving highly connected proteins.

We then examined the interaction networks resulting from the first screenings according to the PBS categories and literature data and conducted selection of secondary and tertiary baits. First, we isolated 54 prey proteins of interest to design 57 new bait domains for a second round of screenings and then, we used the resulting $\mathrm{Y} 2 \mathrm{H}$ network to select 10 additional proteins corresponding to 11 baits for a third and last round of screening. Two of the chosen baits showed autoactivation capacities (CMYA5 $5_{\mathrm{Q} 3501-\mathrm{K} 4069}$ and RCOR3 $\mathrm{M1}_{\mathrm{M}-\mathrm{L} 296}$ fragments) and were therefore discarded. Overall, we successfully carried out 87 large-scale $\mathrm{Y} 2 \mathrm{H}$ screenings using 76 different bait proteins. The comprehensive set of baits is listed in Additional file 2: Table S2.

\section{General properties of the $\mathrm{Y} 2 \mathrm{H}$ interaction map}

The 87 screens led to the identification of 1625 SIDs. On average, each $\mathrm{Y} 2 \mathrm{H}$ assay yielded 18.7 SIDs with a range of 1 to 107. This corresponds to a mean of 19.7
PPIs per bait protein with 53 pairs of connected proteins showing two or more SIDs on the prey protein (Table 2). Performing secondary and tertiary screenings theoretically allowed us to identify reciprocal hits between the secondary/tertiary bait protein and the protein it was originally found to interact with. Practically, reciprocal screenings were not symmetrical because the prey and bait domains were expressed as fusion with a DNAbinding or GAL4-activating domain, respectively and because domains first identified as prey, and then chosen as bait, were rarely identical for practical cloning reasons. Nevertheless, we were able to reciprocally detect nine interactions (Additional file 1: Table S1) that were automatically grouped into the PBS-A category.

Results from all individual $\mathrm{Y} 2 \mathrm{H}$ screenings were assembled in a single network of 1492 PPIs connecting 1018 proteins (LGMD-centered network, Figure 1). Topological analysis of this network using the Cytoscape platform (www.cytoscape.org) revealed an average connectivity of 2.88 partners per connected protein. The mean shortest path length between any of two proteins was calculated as 3.75 . The set of interactions presenting the highest level of confidence consists in the PPIs ranked into the $\mathrm{PBS}-\mathrm{A},-\mathrm{B}$ or $-\mathrm{C}$ categories and 


\begin{tabular}{|c|c|c|}
\hline & Value & $\begin{array}{l}\text { Range, } \\
\text { percentage }\end{array}$ \\
\hline $\begin{array}{l}\text { Total number of bait domains/ } \\
\text { proteins }\end{array}$ & $87 / 76$ & \\
\hline Average number of tested diploids & $\begin{array}{l}10310^{6} \text { per } \\
\text { screen }\end{array}$ & $\begin{array}{l}2010^{6}-203 \\
10^{6}\end{array}$ \\
\hline $\begin{array}{l}\text { Average number of processed } \\
\text { positive clones }\end{array}$ & $\begin{array}{l}155 \text { per bait } \\
\text { domain }\end{array}$ & $3-686$ \\
\hline Total number of proteins/PPIs & 1018/1492 & \\
\hline PBS-A & 234 & $15.7 \%$ \\
\hline PBS-B & 147 & $9.8 \%$ \\
\hline PBS-C & 110 & $7.4 \%$ \\
\hline PBS-D & 800 & $53.6 \%$ \\
\hline PBS-E & 201 & $13.5 \%$ \\
\hline $\begin{array}{l}\text { Average number of SIDs per } \\
\text { bait domain }\end{array}$ & 18.7 & $1-107$ \\
\hline $\begin{array}{l}\text { Average number of partners per } \\
\text { prey protein }\end{array}$ & 1.5 & $1-28$ \\
\hline Mean connectivity & 2.88 & \\
\hline $\begin{array}{l}\text { Total number/average size of } \\
\text { identified SIDs }\end{array}$ & 1625 /231aa & \\
\hline
\end{tabular}

comprises $32.9 \%$ of all PPIs, a figure similar to the one found by [14]. This set defined the most probable truepositive interactions and comprised 491 PPIs consisting of 376 interacting proteins. Among the other remaining interactions, $53.6 \%$ and $13.5 \%$ were classified in the PBS$\mathrm{D}$ and $-\mathrm{E}$ categories, respectively. The high level of PBSD interactions reflects the high complexity of the screened library and the fact that it is screened to saturation but should be considered as putative. General properties of the LGMD-centered interaction map are summarized in Table 2 and the complete list of PPIs along with their PBS category is available in Additional file 1: Table S1.

\section{Examination of interaction domains}

Contrasting with other $\mathrm{Y} 2 \mathrm{H}$ approaches in which bait proteins are tested against full-length prey libraries [1,2], our approach was domain-oriented since the prey library was generated as random-primed cDNA with an average fragment size of 800-1000 bp and since some of the baits consisted of selected domains. For each prey protein involved in a PPI ranked in the PBS-A, $-\mathrm{B}$ or $-\mathrm{C}$ categories, we analysed its $\operatorname{SID}(\mathrm{s})$ and compared their coordinates with predicted InterPro domains to detect SIDs that were included in or included a documented domain. In total, 601 SIDs were computed and showed an average size of 176 aa (Additional file 3: Table S3). This analysis resulted in the identification of 311 distinct SIDs that could be unambiguously associated with one or more InterPro domains either because they were fully included within one such domain (82 SIDs) or because they fully included one or several of them (248 SIDs). The most frequent InterPro domains found within SIDs (in more than $4 \%$ of the SIDs; Table 3) were Immunoglobulin-like domains (IPR003599; IPR003598 and IPR007110), Zinc Finger C2H2 (IPR007087), Fibronectin type III domain (IPR003961), Ankyrin repeat (IPR002110) and Nebulin motif (IPR000900). Finally, we compared the occurrence of InterPro domains fully included within the set of analyzed SIDs with their occurrence in the full human proteome according to [25]. Interestingly, three among the five top-ranking domains in Human were also present with a high frequency in the analyzed dataset although with different ranks and percentages (Table 3). The other two most frequent domains in our subset (Ankyrin repeat and Nebulin motif) were largely over-represented ( $\mathrm{p}$-value $=7.62 \mathrm{e}-16$ and $<2.81 \mathrm{e}-20$, respectively) compared to the global human proteome whereas the three and four top-ranking domains in Human (EGF/laminin and P-loop nucleotide triphosphate hydrolase) were scarce in the analyzed SIDs.

\section{Quality assessment of the $\mathrm{Y} 2 \mathrm{H}$ network}

For all large-scale studies aimed at experimentally identifying molecular interactions, the technical false positive rate is of special concern. We addressed this problem with the assignment of a PBS category. Furthermore, literature mining, cross-validation assays, and functional correlations were used to further estimate the overall solidity of the network.

\section{Comparison with known interactions data}

We first compared our LGMD-centered Y2H dataset with literature-curated interactions between the corresponding mammalian proteins that are referenced in the molecular interaction database iRefWeb (http:// wodaklab.org/iRefWeb/ [22]). This web interface reports data on PPIs consolidated from major public databases such as IntAct, BIND or HPRD. Among our PPIs, 72 were already reported, representing $4.8 \%$ of our map (Additional file 1: Table S1), a figure slightly above others found in large-scale $\mathrm{Y} 2 \mathrm{H}$ studies aimed at exploring the human interactome (3.4\% [2] and 3.8\% [6]). We observed a strong enrichment for literature-based interactions within the PBS-A category for which $18 \%$ of PPI (43) correspond to previously known interactions ( $\mathrm{p}$-value $=1.54 \mathrm{e}-18)$, thus confirming the correlation between the PBS and the biological significance of the interaction.

\section{Experimental cross-validations}

To provide more evidence that the identified physical interactions are genuine, we applied different experimental 


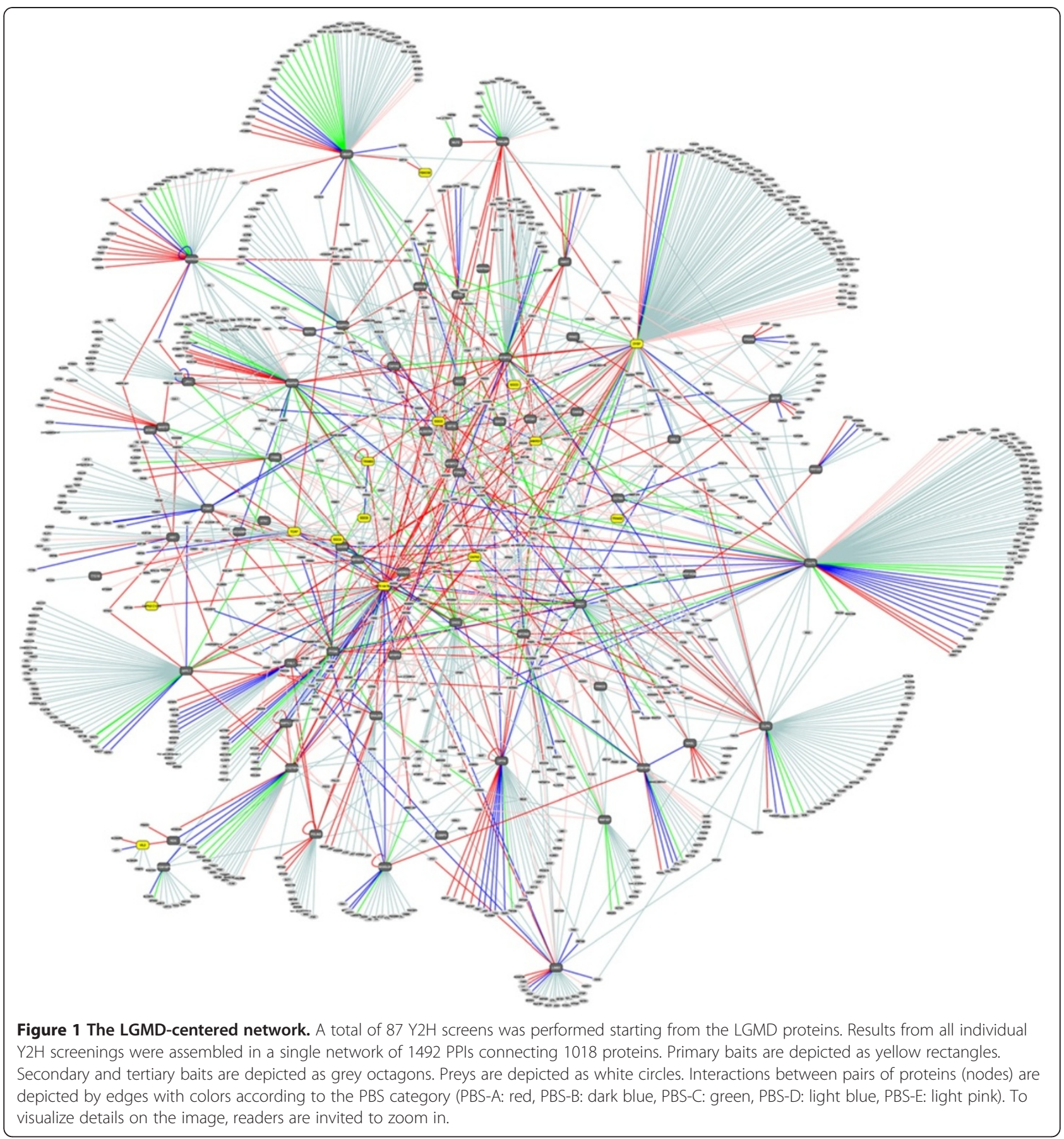

techniques that enable detection and visualization of protein interaction. We first tested a subset of PPIs for the baits: abl-interactor 1 (ABI1), DYSF, enolase 1 (ENO1), SNAP-associated protein (SNAPIN) and telethonin (TCAP), using co-immunoprecipitation in murine R9 myogenic cells at myoblast or myotube stages or in gastrocnemius mouse muscle. A large proportion of the 42 interactions tested were technically inconclusive due in part to the quality of the available antibodies or stickiness of both proteins. Among the 14 technically conclusive, 9
(64\%) were confirmed as positive by the co-immunoprecipitation assays (1 PPI for ABI1, 5 for DYSF, 1 for SNAPIN and 2 for TCAP; Figure 2 and Additional file 1: Table S1). Several interesting outcomes emerge from these validations that reinforce and extend the knowledge we have on LGMD proteins or open new directions for specific areas of research. For example, the interaction between DYSF, the LGMD2B protein, and SNAPIN, a component of the SNARE complex that is required for vesicle fusion [26] is appealing with regard to the role of 
Table 3 Most frequent domains in the LGMD-centered dataset and representativeness in the human proteome

\begin{tabular}{lcc}
\hline Domains & \% in the total of SID & $\begin{array}{c}\text { \% in the human proteome } \\
\text { (according to Müller et al., Genome Res 2002) }\end{array}$ \\
\hline Immunoglobulin (IPR003599; IPR003598; IPR007110) & $22.5 \%(n=141)$ & $4.2 \%(n=1214 ; r=2)$ \\
Zinc finger, C2H2-type (IPR007087) & $12.8 \%(n=80)$ & $17.6 \%(n=5092 ; r=1)$ \\
Fibronectin type III (IPR003961) & $9.63 \%(n=60)$ & $2.9 \%(n=842 ; r=5)$ \\
Ankyrin repeat (IPR002110) & $5.3 \%(n=33)$ & $0.9 \%(n=278 ; r=14)$ \\
Nebulin 35 residue motif (IPR000900) & $4.5 \%(n=28)$ & $n . i$.
\end{tabular}

(n.i.= not indicated; $n=$ number of occurrences; $r=$ rank in the human proteome).

DYSF in membrane repair [27]. The positive interaction between kinectin (KTN1), a kinesin anchoring protein that modulates the endoplasmic reticulum interaction with the microtubule and that is known to interact with Rho GTPases [28], and ABI1, an adaptor protein involved in the transduction of signals from Ras to Rac and the regulation of actin polymerization [29], suggests a role for this interaction in the regulation of the muscle cytoskeletal remodeling.

We then investigated intracellular co-localization on normal human muscle sections of one particular bait, DYSF, with its $\mathrm{Y} 2 \mathrm{H}$ partners for which quality antibody was available for immuno-fluorescence assays. Among the 14 PPIs investigated, five showed no apparent colocalization while the others demonstrated different degrees of co-localization at the sarcolemma and/or in the cytoplasm (Figure 3). Analysis of Pearson's coefficient showed that DYSF labeling presented a strong correlation with the cardiomyopathy-associated protein 5 (CMYA5, 0.567), DES (0.656), filamin-C (FLNC, 0.521), kinesin family member 1B (KIF1B, 0.566) and optineurin (OPTN, 0.733), a medium correlation with Alstrom syndrome protein 1 (ALMS1, 0.402), diacylglycerol kinase delta (DGKD, 0.309), and nebulin (NEB, 0.332) and a low correlation with SNAPIN (0.12).

We also visualized the sub-cellular localization of the interactions using a Duolink proximity ligation assay (PLA) for 44 PPIs. The Duolink technology is a combination of immunohistochemistry and rolling circle amplification and is based on the use of bifunctional probes consisting of a secondary antibody attached to a synthetic oligonucleotide. It generates a quantifiable signal indicative of close proximity $(<40 \mathrm{~nm})$ between two antigens. For our purpose, primary antibodies against five different baits [DYSF, OPTN, $\gamma$-sarcoglycan (SGCG), adaptor protein containing $\mathrm{PH}$ domain, $\mathrm{PTB}$ domain and leucine zipper motif 1 (APPL1) and myomesin2 (MYOM2)] and some of their partners identified in our $\mathrm{Y} 2 \mathrm{H}$ screenings (18 interacting partners for DYSF, 10 for OPTN, 3 for SGCG, 3 for APPL1 and 5 for MYOM2)

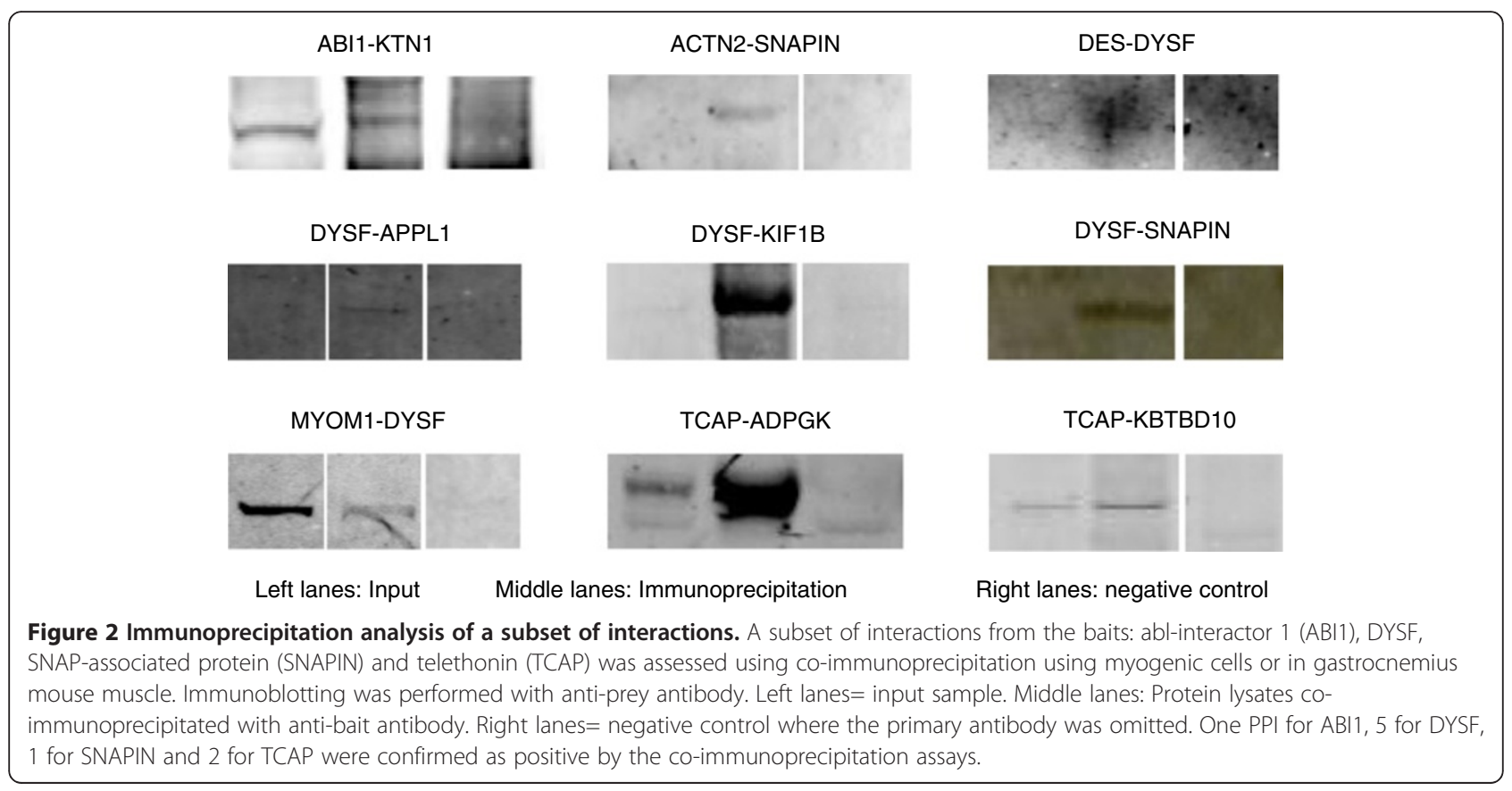




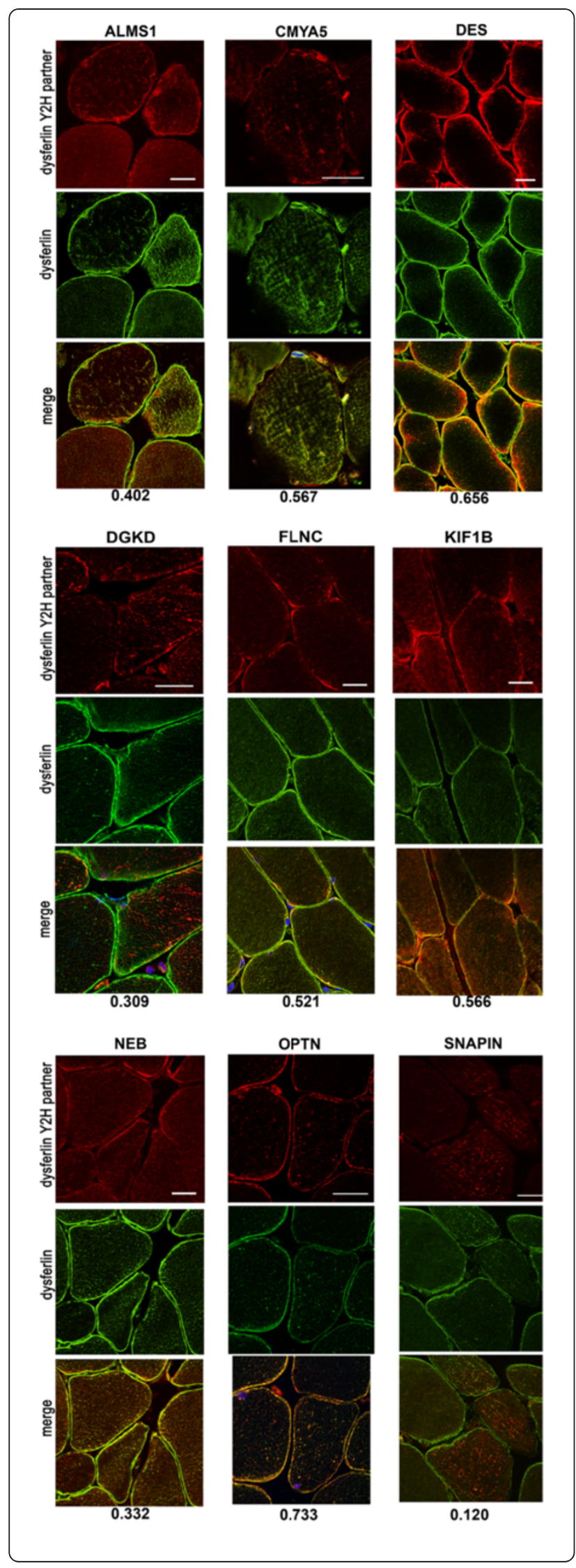

Figure 3 Co-localization analyses of dysferlin and its partners. Double immunostaining for DYSF (Alexa488, green) and its $\mathrm{Y}_{2} \mathrm{H}$ partners (Alexa594, red) in cross human muscle cryosections by alphabetic order. The images were taken with a 40x objective for DES, FLNC, and NEB and a 63x for OPTN, KIF1B, DGKD, SNAPIN, and CMYA5. Scale bars $=20 \mu \mathrm{m}$. The Pearson's coefficient is indicated under the merge image for each PPI. As expected, DYSF showed a membrane-associated pattern and a reticular cytoplasmic pattern corresponding to T-tubules on transversal section. Depending on the tested protein, the partner pattern is variable.

were used to obtain a PLA "PPI signal". In a separate experiment, only the primary antibody of the prey was used to generate the "Prey signal". In this way, the cellular site(s) where the binary interactions were occurring could be visualized. Among the 44 tested interactions, 34 showed a "PPI signal" with various proportions between membrane and cytoplasm. An image analysis was performed to calculate the ratio of the "PPI signal" compared to the "Prey signal" separately at the membrane or cytoplasm locations (Figure 4A). Two examples of DYSF interactions are depicted in Figure 4B. The single protein detection of DYSF revealed as expected the presence of the protein at the membrane and at the Ttubule network within the cell (Figure 4B). The APPL1 protein seems almost entirely engaged in the interaction with DYSF both at the membrane and cytoplasm locations. In contrast, DES/DYSF association was visualized only at membrane level.

In total and among the 54 PPIs investigated using these three different techniques, 40 interactions were considered as relevant by at least one technique (74\%) with 20 of them from the PBS-A, $-\mathrm{B}$, or $-\mathrm{C}$ categories and the others from the PBS-D category. The 26\% remaining interactions identified by our $\mathrm{Y} 2 \mathrm{H}$ screens that could not be experimentally cross-validated in our hands consist in 3, 3, 2 and 5 interactions classified in the PBS-A to $-\mathrm{D}$ categories, respectively.

\section{Examination of GO annotations}

We performed computational analyses using the Database for Annotation, Visualization, and Integrated Discovery (DAVID) web-resource (http://david.abcc.ncifcrf. gov/) to detect pairs of interacting proteins known to participate in similar biological processes (BP), to be part of the same cell components (CC) or to perform similar molecular functions (MF). In the LGMD-centered network, 84,72 and $82 \%$ of proteins were annotated for the BP, CC and MF GO categories, respectively. Among the annotated proteins, we identified 449 pairs of proteins $(30 \%)$ that share a GO annotation, including 168 pairs within the PBS-A, $-\mathrm{B}$, or $-\mathrm{C}$ categories (34\%; Additional file 1: Table S1). More precisely, among PPIs from the PBS-A, $-\mathrm{B}$ and $-\mathrm{C}$ categories, a significantly higher number of PPIs with shared BP or CC GO 
A
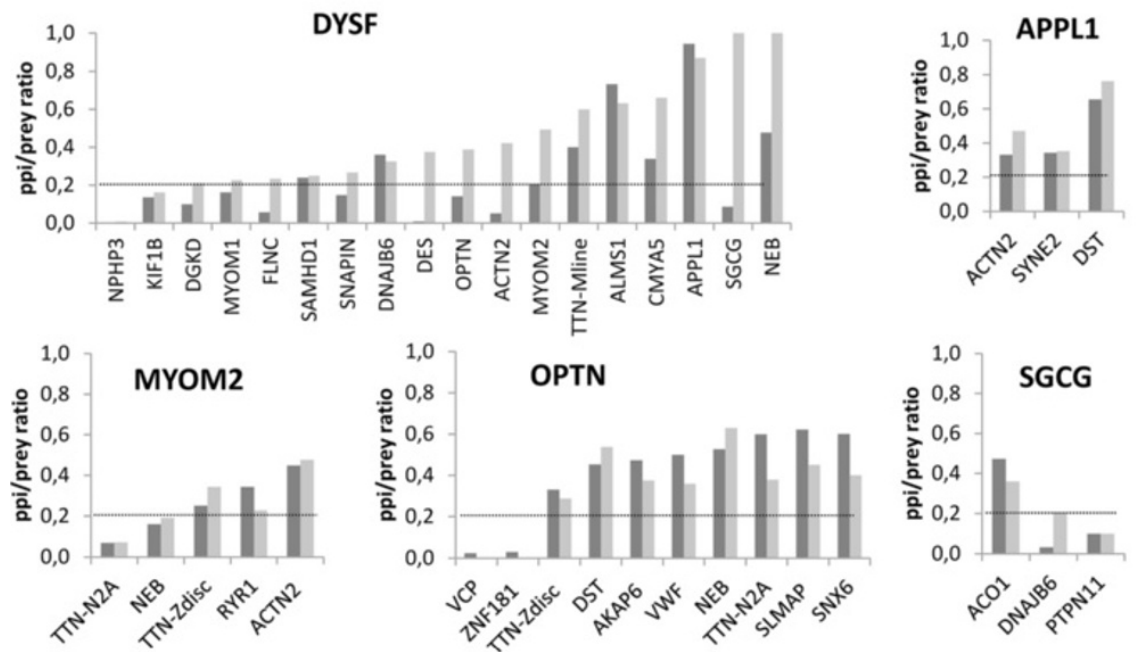

B
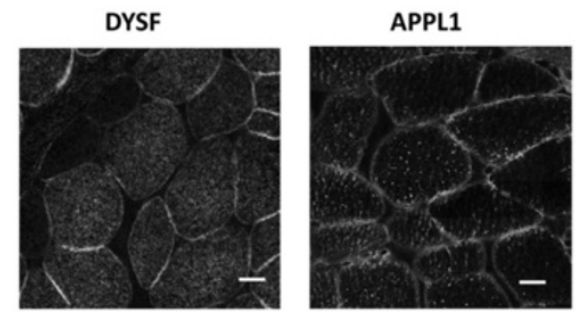

DES

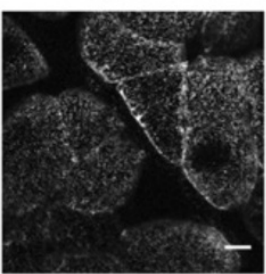

DYSF-DES
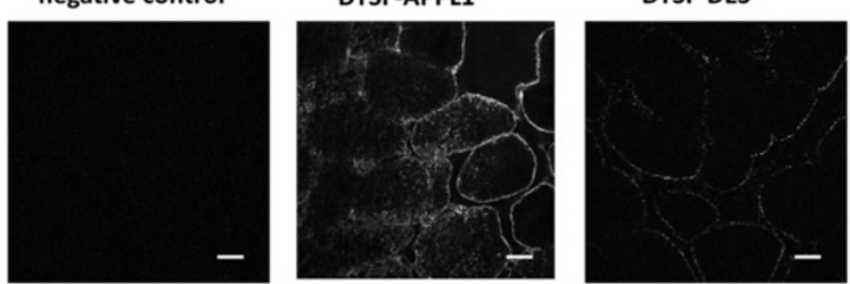

Figure 4 Proximity ligation assays of a subset of interactions. A/ Graphs showing the ratio of the signal for the PPI divided by the signal for the prey protein alone with respect to the ROI corresponding to membrane (light grey) or cytoplasm (dark grey) for the bait proteins DYSF, APPL1, MYOM2, OPTN and SGCG. Prey proteins are named with their gene symbol. The line corresponding to $20 \%$ is indicated for each graph. For technical reasons, in the case of DYSF/SGCG and MYOM2/TTN interactions, the PPI/prey ratio is calculated as the ratio of SGCG and TTN molecules with respect to DYSF and MYOM2 although DYSF and MYOM2 were found as preys of SGCG and TTN baits. For MYOM2 and OPTN, two TTN antibodies were tested with epitopes at the Z-disc and N2A regions, respectively. Among the 44 tested interactions, 34 showed a "PPI signal" with various proportions between membrane and cytoplasm. B/ Representative confocal images of PLA results. Duolink amplifications are visualized by fluorescence (white dots). Upper panels: PLA+/- labeling of single proteins. Left panel = negative control consisting of DYSF with probes corresponding to the PPI for evaluation of background staining. Middle panel: the DYSF-APPL1 interaction signal showed a strong labeling both at the membrane and the cytoplasm. Right panel: the DYSF-DES labeling showed that the signal is mainly located at the membrane. Scale bars $=20 \mu \mathrm{m}$.

annotations compared to the Human proteome were detected (Chi-2 test, $\mathrm{P}<0.05$, Figure 5), confirming again the correlation between the PBS and the biological significance of the interaction.

\section{The NMD proteins in the LGMD-centered network}

We examined our LGMD-centered interactome map to pinpoint interactions involving proteins identified as the genetic cause of one or more hereditary NMDs by searching the OMIM database. We identified 199 proteins of the network that are associated with human monogenic diseases including 77 proteins whose defects have been described as the genetic cause of one or more hereditary NMD (Additional file 4: Table S4) . Among this last group, 43 proteins correspond to myopathies including the recently described DNAJB6 [30], 20 to 


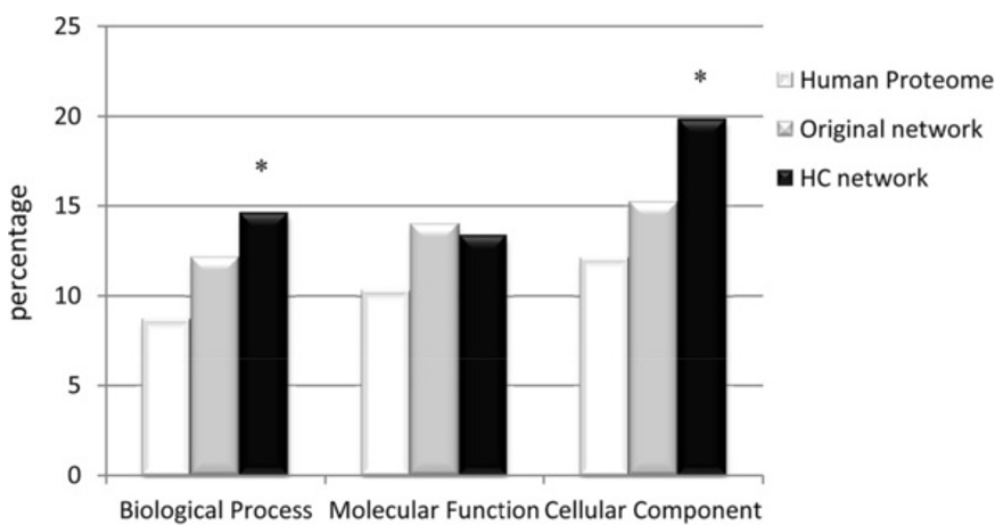

Figure 5 Fraction of proteins pairs sharing a GO annotation cluster. Each bar represents the fraction of proteins pairs that share a cluster within each of the three $\mathrm{GO}$ categories (biological processes, cell components or molecular functions; percentage indicated). Asterisks above the bars indicate an observed value statistically different from the one expected in the Human proteome dataset (Chi-2 test, $\mathrm{P}<0.05)$.

cardiomyopathies, 17 to neuropathies, 8 to metabolic muscle diseases, 3 to excitation abnormalities and 8 to unclassified NMD. From these 199 proteins, it was possible to construct a protein interaction network including 88 proteins in 113 interactions (Additional file 5: Figure S1). No specific repartition of NMD and nonNMD proteins was noticed.

We then examined the sub-network consisting of all the LGMD and other known NMD-causing proteins, and their interacting partners. This sub-network contains 56 proteins whose defects have been described as the genetic cause of one or more hereditary NMD. Aside from the nine LGMD proteins used as baits in our screenings, this network contains three additional LGMD proteins, plectin (LGMD2M), myotillin (LGMD1A) and lamin A/C (LGMD1B). Thirty-five connections of LGMD-proteins with other NMD-causing proteins were identified with 28 novel and direct interactions. In particular, this analysis revealed three novel direct interactions linking TTN, DYSF and SGCG (Figure 6A). We examined the type of diseases the non-LGMD proteins are responsible for and found that proteins involved in congenital and metabolic myopathies as well as other neuromuscular disorders were significantly over-represented $(\mathrm{p}$-value $=1.04 \mathrm{e}-05,0.025$ and 0.040 , respectively). It should be noted that the proteins involved in the metabolic myopathies glycogen storage disease type 13 (GSD13) and glycogen storage disease type 5 (GSD5), that are $\beta$-enolase (ENO3) and the muscle form of glycogen phosphorylase (PYGM), respectively, present a high number of physical interactions with the LGMD proteins group. Interestingly, we observed the existence of a dense network around the group of LGMDcausing proteins with many of them linked by paths of three nodes or shorter (Figure 6B). It can be noted that among the hub proteins in this network are proteins located at three important positions of the sarcomere: actinin $\alpha 2$ (ACTN2) for the Z-disc, myosin-binding protein $\mathrm{C}$, slow-type (MYBPC1) and MYBPC2 for the N2A-line and MYOM2 for the M-line.

Considering that the LGMD-centered interactome is strongly enriched in known NMD-causing proteins, we expect that some yet-uncharacterized NMD-causing proteins are part of our interaction network. We examined in more detail the candidate genes for the orphan LGMDs. In addition to the causative genes for 4 dominant and 17 recessive LGMD forms that are known so far, genetic linkage analyses have been used to map loci for three new LGMDs. These orphan LGMD loci contain between 13 and 45 genes, with a total of 2 genes encoding proteins of the LGMD-centered interactome. Consequently, FLNC and protein transport protein Sec31A (SEC31A) were isolated as unique candidates for LGMD1F and LGMD1G, respectively. No gene from the LGMD-centered interactome was found within the LGMD1E locus.

\section{Properties of the LGMD-centered network and biological functions of the proteins}

To investigate which biological functions are associated with the LGMD-centered network, we performed a GO term enrichment analysis. In this analysis, we compared the LGMD dataset to a high-confidence (HC) network encompassing the most meaningful PPIs as defined with combined results from the experimental and computational analyses described above and a literature-based network.

The HC dataset was built by extracting from the LGMD-centered map the 491 PPIs classified in the PBS$A$, $-\mathrm{B}$, or $-\mathrm{C}$ categories plus PPIs from the PBS-D and -E categories for which we obtained additional evidence of the interaction. First, we added the 22 of our $\mathrm{Y} 2 \mathrm{H}$ interactions from the PBS-D or -E categories that have been described in other studies (Additional file 1: Table S1). Second, PPIs from the PBS-D or -E categories were 


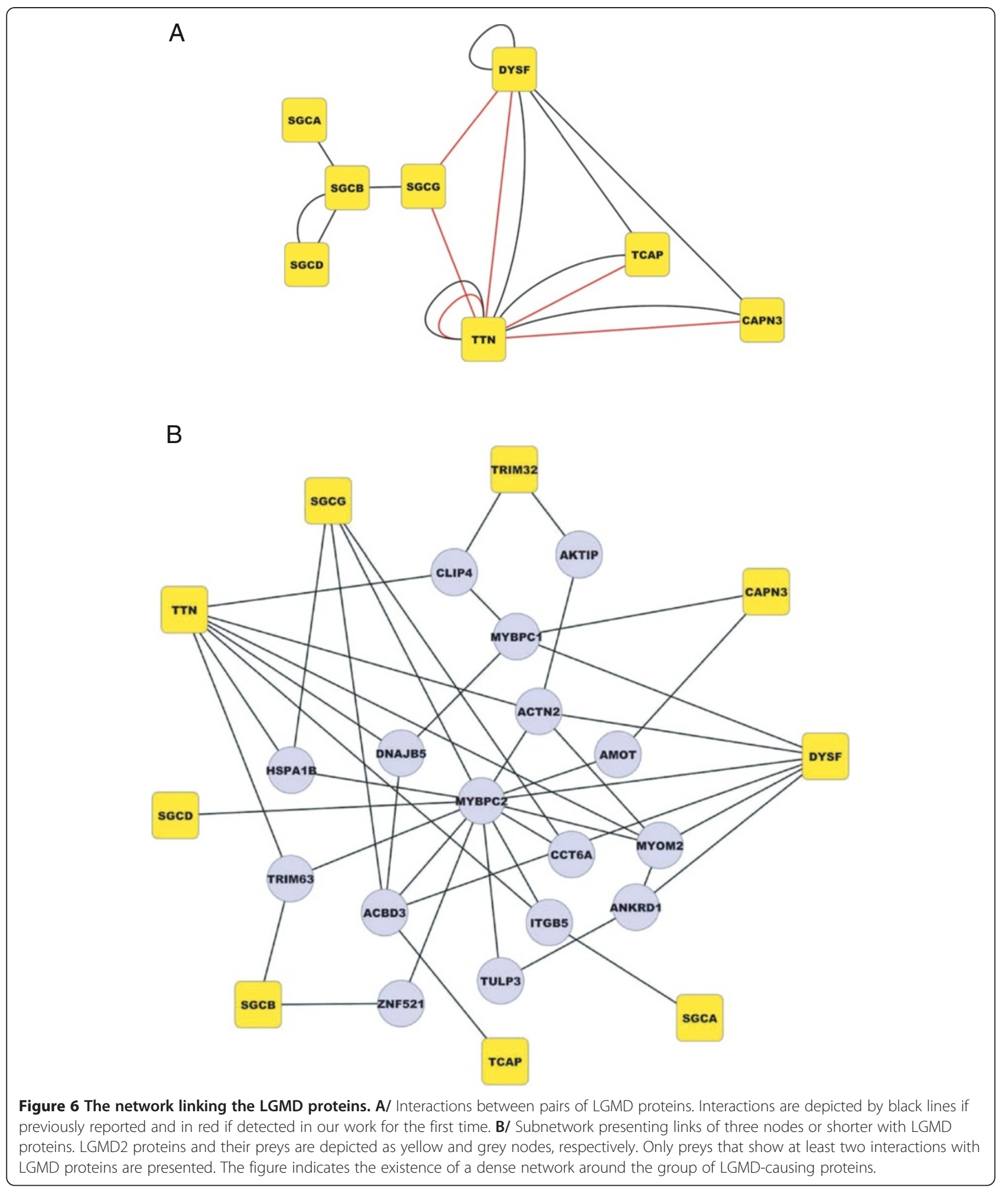

added to the $\mathrm{HC}$ dataset if experimental results from coimmunoprecipitation, immunofluorescence or PLA as described above confirmed the $\mathrm{Y} 2 \mathrm{H}$ interaction. In total, 20 experimentally cross-validated interactions from the PBS-D category were included in the HC dataset. Third, were also included in the HC network, 107 pairs of interacting proteins from the PBS-D or -E categories that either share a BP GO annotation or share a CC and a MF $\mathrm{GO}$ annotation. Finally, since our dataset unraveled a high average level of relationship between NMD proteins, 65 
PPIs in the PBS-D and -E categories and involving a NMD prey protein were added to the $\mathrm{HC}$ network. The resulting $\mathrm{HC}$ dataset consists of 497 proteins and 705 PPIs (Additional file 1: Table S1) and includes 174 and 40 PPIs from the PBS-D and -E categories, respectively.

The literature-based dataset was constructed by combining all direct protein-protein interactions reported in the literature for proteins of the HC dataset or LGMD-causing proteins that were not included in our initial set of baits. It is interesting to note that only two interactions for the five LGMD glycosyltransferases have been identified in previous reports [between Fukutin and protein O-linked-mannose beta-1,2-N-acetylglucosaminyltransferase 1 (POMGnT1) and between protein O-mannosyl-transferase 1 (POMT1) and 2 (POMT2)]. Since the glycosyltransferases are all described or predicted to be single or multipass membrane proteins, the rarity of PPI and their absence in our LGMDcentered network are possibly explained, since identification of interactions in the vicinity of membranes has proven to be difficult with the $\mathrm{Y} 2 \mathrm{H}$ method. We searched for previously published experimental binary and direct interactions using the iRefWeb interface and identified 2675 direct binary interactions (Additional file 6: Table S5). This literaturebased network consists of 2239 proteins and 3304 PPIs.

Characteristics of the three networks were analyzed (Table 4) [31]) and showed a connectivity of 0.022, 0.024 and 0.041 for the original and HC LGMD-centered networks and the literature-based network, with the proteins being associated with 19.7, 9.7 and 25.4 PPIs on average, respectively. Network heterogeneity, a parameter that reflects the tendency of a network to contain hub nodes was $2.895,1.964$ and 3.833 for the original and HC LGMD-centered networks and the literaturebased network, respectively.

Using the DAVID web-resource and an appropriate level of abstraction of the annotations, we performed a GO term enrichment analysis of the three datasets in order to check if they presented any specificity in terms of biological process, cellular localization or molecular function of their proteins as compared to the human proteome (Figure 7). Not unexpectedly, they show an over-representation of proteins involved in muscle biology, even quite exclusively for both the original and $\mathrm{HC}$ LGMD-centered networks that share very similar

Table 4 Comparison of the three networks

\begin{tabular}{llll}
\hline & Original & HC & $\begin{array}{l}\text { Literature- } \\
\text { based }\end{array}$ \\
\hline Number of proteins & 1018 & 497 & 2239 \\
Number of PPIs & 1492 & 705 & 3304 \\
$\begin{array}{l}\text { Average number of partners per bait } \\
\text { protein }\end{array}$ & 19.7 & 9.7 & 25.4 \\
Mean connectivity & 0.024 & 0.022 & 0.041 \\
\hline
\end{tabular}

HC: high-confidence; PPI: protein-protein interaction. profiles (Figure 7). The biological processes with most striking enrichments in all datasets are muscle contraction, cytoskeleton organization and muscle organ development. The cellular components with the highest representation include contractile fibers and cytoskeleton. The enriched molecular functions are cytoskeletal protein binding and structural constituents of muscle protein. In addition to these terms, the literature-based network shows additional terms with a significant enrichment of proteins involved in cell cycle and death, localized in the cytosol and the nucleus and with broader molecular functions (binding of enzyme, transcription factor or ATP and protein kinase).

A table explaining the component of all the supplements is added as Table 5.

\section{Discussion}

Our $\mathrm{Y} 2 \mathrm{H}$ experimental strategy was a large-scale protein domain-based approach using a methodology that has been previously successfully implemented [14]. As compared to approaches where baits are screened against full-length prey proteins, a domain-based approach offers several advantages including the possibility of narrowing down interaction domains and of reducing the false negative discovery rate by allowing a more efficient folding of domains [32]. A similar approach used in the field of ataxia identified interactions that were missed in a previous full-length protein-based approach [33]. Interestingly, this domain-based approach enabled us to analyze possible bias in interacting domains. We found that the three most frequent domains present in the SIDs (Immunoglobulin-like, Zinc Finger and Fibronectin domains) are also amongst the most frequent ones in the human proteome, emphasizing how important biomolecular interactions are for cellular processes. Two other domains (Ankyrin repeat and Nebulin motif) were frequently reported in the SIDs whereas they are rare in the human proteome. These domains were established as useful for meeting the demands of skeletal muscle physiology and constraints [34,35]. Auto-binding capacities of ankyrin repeats lead to mechanical-resistant dimers and nebulin-like domains play a role in the regulation of muscle contraction, especially through their interaction with actin and the thin filament. On the other hand, two domains frequently found in the human proteome (EGF-like and P-loop domains) are underrepresented in our set of interacting domains. This observation could be explained either by the function of the domain; the P-loop motif is known to be involved in hydrolysis of ATP and GTP but not in protein-protein interaction; or by a technical bias as in the case of the EGF-like domain which principally serves as an interacting domain within extracellular protein modules. The underrepresentation of this last domain is 

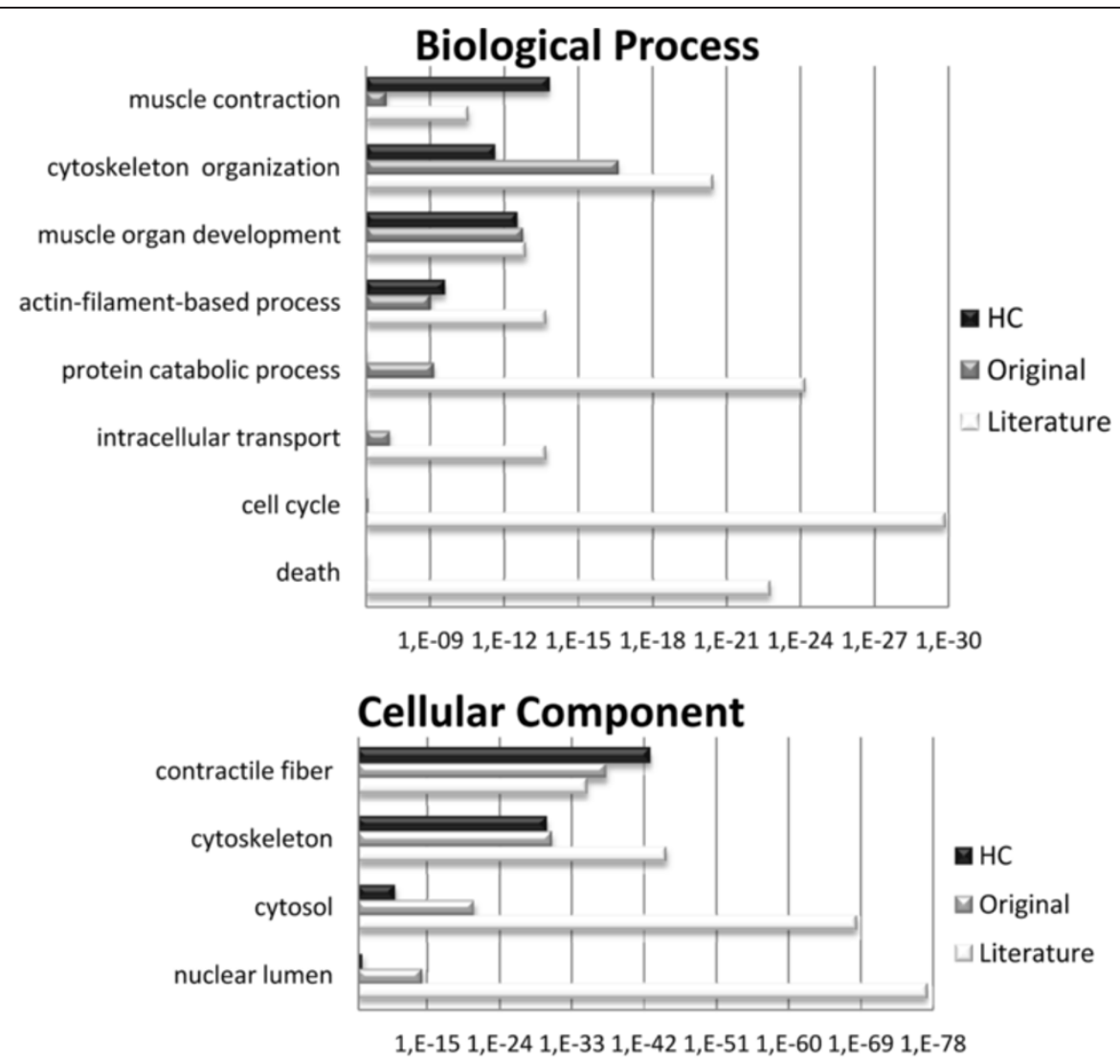

\section{Molecular Function}

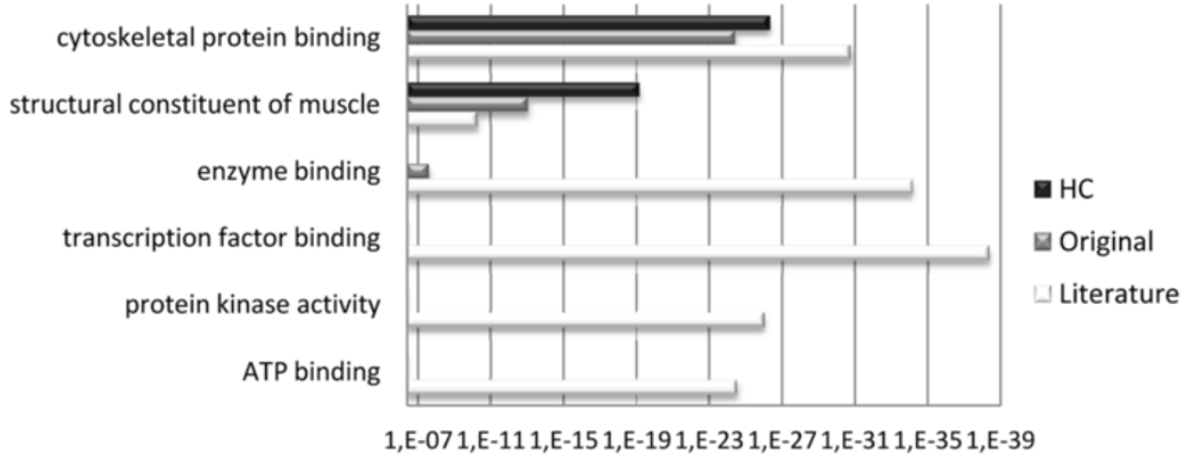

Figure $7 \mathrm{GO}$ enrichment analysis of the three datasets compared to the human proteome. A GO term enrichment analysis of the three datasets (LGMD-centred, HC and literature-enriched datasets) was performed using the DAVID web-resource and an appropriate level of abstraction of the annotations, The bar charts depict enriched GO terms of the three datasets compared to the human proteome in the three branches of the GO structure as a function of the EASE score.

probably related to the fact that the LGMD proteins are intracellular proteins with the exception of sarcoglycans but for which the intracellular domain was selected for the $\mathrm{Y} 2 \mathrm{H}$ screening.

The utility of any network obviously depends on the quality of the data. Our approach resides in the exploitation of random-primed cDNA libraries constructed from human skeletal muscle poly(A) RNA. It minimizes the risk of detecting interactions between proteins that would not be co-expressed in the muscle tissue and therefore should reduce the number of biological false positives. In addition, assignment of a PBS score using a statistical method allowed us to classify each PPI identified in our $\mathrm{Y} 2 \mathrm{H}$ assays into five predictive categories, ranging from the most reliable interactions to possible technical false positives. The PBS score was previously demonstrated to successfully predict the reliability of a PPI on a subset of $\mathrm{Y} 2 \mathrm{H}$ interactions where the authors experimentally 
Table 5 Additional file contents

\begin{tabular}{ll}
\hline Additional file & Contents \\
\hline Additional file 1: Table S1 & $\begin{array}{l}\text { gives a comprehensive list of all the } \\
\text { identified PPI }\end{array}$ \\
Additional file 2: Table S2 & $\begin{array}{l}\text { gives comprehensive information } \\
\text { about the baits and Y2H screenings }\end{array}$ \\
Additional file 3: Table S3 & $\begin{array}{l}\text { gives the list of all the SIDs } \\
\text { presents the Human diseases associated } \\
\text { Additional file 4: Table S4 }\end{array}$ \\
Additional file 5: Figure S1 & $\begin{array}{l}\text { presents a sub-network composed of } \\
\text { the disease-related proteins only } \\
\text { gives the list of the PPIs for the }\end{array}$ \\
Additional file 6: Table S5 & literature-based network
\end{tabular}

PPI: protein-protein interaction; SID: selected interacting domain; $Y 2 H$ : yeasttwo hybrid.

confirmed $79 \%$ of all PPIs from the PBS-A, -B or -C categories by pull-down or co-immunoprecipitation assays [14]. The PBS takes into account both local parameters, such as the number of identical or independent fragments found for each partner and global information derived from the entire network such as reciprocal interactions, highly connected domains etc... Our screens identified a high percentage of the PBS-D interactions (53.6\%) which reflects the high complexity of the constructed muscle library and the fact that this library is screened to saturation. As a consequence, the rate of false negatives should be extremely low, as even rare transcripts in the library, or weak or transient interactions, could be detected. In line with the notion that PBS-D interactions might be more difficult to detect, a higher proportion of interactions from the $\mathrm{ABC}$ categories than from the $\mathrm{D}$ category can be confirmed by other methods, such as coimmunoprecipitation or pull-down [14]. However, they could also represent false positive interactions and should be considered with caution. Finally, over 210 publications (full list available http://www.hybrigenics-services.com/ publications/index/list). Of note, $27 \%$ of the publications reporting the functional validation of protein interactions identified using the same $\mathrm{Y} 2 \mathrm{H}$ methodology correspond to the validation of a $\mathrm{D}$ interaction. This indicates that a significant proportion of such low confidence $\mathrm{Y} 2 \mathrm{H}$ interactions are functionally relevant.

In the present study, we further confirmed the relevance of the PBS score with computational and experimental evidence. First, nearly a fifth of the PBS-A PPIs were found to have been previously reported whereas this figure falls to less than $5 \%$ when considering the whole interactome. Second, co-immunoprecipitation assays confirmed as positive 11 out of 16 technically conclusive tests (69\%) and PLA validated 34 of the 44 tested interactions (77\%). Although there is often limited overlap between studies [2,36], probably in part because of the spatial and temporal aspects of the proteome interaction, we compared our results obtained for DYSF with a recent study that analyzed the composition of DYSF complexes in cultured myoblasts, myotubes and skeletal muscle tissues by mass spectrometry and bioinformatics methods [37]. Interestingly, 19 (14\%) of the 136 DYSF interactions identified by our large-scale $\mathrm{Y} 2 \mathrm{H}$ screening were also found in the de Moree's study, a ratio that is a little higher than what is usually observed [3]. In contrast, only 3 interactions (TTN, ACTN2 and DES) were found in a study based on Fisher' method [38] and none in another in silico study [39].

Remarkably, results of our $\mathrm{Y} 2 \mathrm{H}$ screens led to a single connected network where the different LGMD proteins are highly connected. The strong inter-connectivity between LGMD proteins is illustrated by a high number of direct interactions. This was quite surprising since, even if the different LGMD forms share seemingly close clinical phenotypes, the LGMD-causing proteins have been described to have quite diverse locations and biological functions. In addition to identifying a remarkable number of direct interactions between LGMD proteins, mining the data revealed that LGMD proteins belong to a highly connected network of interacting proteins with, in particular, the sarcomeric proteins ACTN2, MYBPC1, MYOM1 and MYOM2 identified as hub proteins sharing the highest number of links with the LGMD proteins. Interestingly, these proteins are structural proteins located at key places on the sarcomere, the Z-disc, the N2A-line and the Mband. GO analyses further support the crucial place of the cytoskeleton in the connections between LGMD proteins. Taken together, these data suggest that common molecular mechanisms underlie the pathogenesis of these diseases and highlight the sarcomere as an important platform for skeletal muscle homeostasis and myofiber survival.

We expect that our interaction map can serve as a new tool to accelerate discovery of the causative mutated genes for orphan LGMDs or for other orphan NMDs already or not yet described as well as to identify modifier genes. Examination of the chromosomal location of all the genes coding for the proteins that are part of our original interactome map revealed putative candidates for orphan NMDs of which FLN and, SEC31A appeared to be of particular interest for LGMD1F and LGMD1G, respectively. Defects in the FLNC gene coding for an actin-binding protein, are already known to cause myofibrillar myopathies [40] but the gene was supposedly excluded as being involved in the pathogenesis of LGMD1F [41]. Nevertheless, non-coding sequences were not fully investigated for pathogenic mutations and, as mentioned by the authors, the gene remains a possible candidate. The SEC31A gene coding for a component of a protein complex responsible for vesicle budding from the endoplasmic reticulum [42], has not been associated with any disease. It is an interesting candidate since LGMD1G is associated with progressive limitation of fingers and toes flexion [43] and 
SEC31A has been linked to collagen secretion [44]. In addition to the LGMD proteins, there is a high proportion of proteins involved in congenital and metabolic myopathies in our network, therefore it is very likely that causative genes for genetically uncharacterized forms of these two groups of diseases lie within our interactome.

An interesting outcome of our study is to provide new PPIs that further support and extend previous findings and pinpoint new pathways of interest that could be affected in LGMD. For example, two novel interactions of calpain 3 (CAPN3) with, ring finger protein 167 (RNF167), an E3 ubiquitin-protein ligase, and the proteasome maturation factor (POMP) are of particular interest considering a previous report indicating that CAPN3 acts upstream of the ubiquitin-proteasome system [45]. For DYSF, a number of new interacting partners can be categorized in three different cellular processes: endocytosis, microtubule-related transport and regulation of gene expression. The first two pathways fit well with previous knowledge about DYSF functions but, interestingly, the third pathway indicates a new possible role for this protein. Another interesting finding in view of the fact that the pathogenesis of SGCG deficiency does not seem strictly related to membrane stability $[46,47]$, is the possible relationship of this protein with energy controlling pathways since interaction with proteins involved in glycolysis or glycogenolysis (enolases 1 and 3 and PYGM) or in the TCA cycle (SUCLG2, ACO1) was identified. Finally, several partners detected for TCAP suggest that it may play a role in the maintenance of genome integrity, in accordance to the recent report showing a relationship between TCAP and p53 turnover [48]. These elements provide new avenues to explore for a better understanding of the pathophysiology of the various forms of LGMD.

In conclusion, this study presents new interacting partners for LGMD proteins and other proteins known to be involved in NMD. In this sense, it has the potential to reveal new candidate genes for NMD but also modifiers of the phenotype. This broad dataset should also help to take a step further towards the understanding of skeletal muscle tissue. In particular, it will help to improve our knowledge about the cellular functions and roles of NMD proteins in the muscle cell and about their participation in the diseases they trigger thereby speeding up the identification of putative drug targets.

\section{Additional files}

Additional file 1: Table S1. Comprehensive list of PPI of the LGMDcentered network. Column A: Bait gene symbol; Column B: Gl number of reference for bait sequence (the name of the protein is indicated in Table S1) Additional file 2: Table S2; Columns C-D: HUGO Gene Nomenclature Committee (HGNC) and Uniprot identifiers for the bait (mapped by
DAVID); Column E: Prey gene symbol; Column F: GI number of reference for prey sequence; Column G: Genbank notes for prey protein; Columns $\mathrm{H}-\mathrm{I}$ : HGNC and Uniprot identifiers for the prey (mapped by DAVID); Column J: PBS category (A to E) for the Y2H PPl; Column K: PPI belonging to the HC network (Y=yes); Column L: Reciprocal hits in our experiment (Y=yes); Column M: Pubmed ID for previously described PPI in mammals; Column N: PPI experimentally validated by immunoprecipitation (IP_pos), co-localisation assays (Coloc_pos) or proximity ligation assays (Duolink_pos). The negative results are also indicated (_neg), Columns O$\mathrm{Q}: \mathrm{GO}$ annotation for pairs of proteins sharing a BP, CC and MF cluster, respectively; Column R: Shared NMD associated with the prey protein; Column S: OMIM of the associated NMD.

Additional file 2: Table S2. Comprehensive bait and $\mathrm{Y} 2 \mathrm{H}$ screenings information. a) Interaction was reciprocally found (A<->B) (b) TTN novex3 variant was found as prey.

Additional file 3: Table S3. List of SIDs. Column A: PBS category; Column B: Bait gene symbol; Column C: Bait GI number; Columns D-E: HGNC and Uniprot identifiers for the bait (mapped by DAVID; Column F: Bait coordinates (aa) on the gi sequence translated product; Column G: Prey gene symbol; Column H: Prey GI number; Columns I-J: HGNC and Uniprot identifiers for the prey (mapped by DAVID); Columns K-L: SID coordinate on the prey gi sequence translated product (first and last aa, respectively); Column M-O: For PPIs from the PBS-A, $-B$ and $-C$ categories, Interpro domains including the SID, included within the SID or overlapping with the SID, respectively.

Additional file 4: Table S4. Human diseases associated with the proteins of the network. Column A: Gene symbol; Column B: Gl number of the bait or prey sequence; Column C: Human genetic conditions associated with the protein; Column D: OMIM number ( $N D=$ not

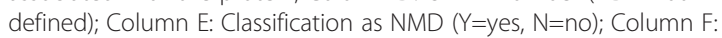
Classification of NMD according to the Neuromuscular Disorders Online Gene Table (http://www.musclegenetable.fr) 1 Muscular dystrophies, 2 Congenital muscular dystrophies, 3 Congenital myopathies, 4 Distal myopathies, 5 Other myopathies, 6 Myotonic syndromes, 7 lon channel muscle diseases, 8 Malignant hyperthermia, 9 Metabolic myopathies, 10 Hereditary cardiomyopathies, 11 Congenital myasthenic syndromes, 12 Spinal muscular atrophies, 13 Hereditary ataxia, 14 Hereditary motor and sensory neuropathies, 15 Hereditary paraplegia, 16 Other NMD.

Additional file 5: Figure S1. Diseases related-protein network. NMDrelated proteins are depicted as blue node ovals and non-NMD-related proteins are depicted as yellow node ovals. Interactions between pairs of disease-related proteins are depicted by edges with colors according to the PBS category (PBS-A: red, PBS-B: dark blue, PBS-C: green, PBS-D: light blue, PBS-E: light pink). Only proteins that show interactions with diseases-related proteins are presented.

Additional file 6: Table S5. List of PPIs for the literature-based network. Column A: Gene symbol for the protein of the interactome; Columns B-C: Gene symbol and HGNC identifier for the interacting partner from the Intact database or the interactome; Column D: Name of the interacting partner.

\section{Abbreviations}

Y2H: Yeast-two hybrid; PPI: Protein-protein interaction; HC: High-confidence; SID: Selected Interacting Domains; PBS: Predicted Biological Score; PLA: Proximity ligation assay; GO: Gene Ontology; BP: Biological processes; CC: Cell components; M F: Molecular functions; NMDs: Neuromuscular Disorders; LGMD: Limb-girdle muscular dystrophy; LGMD2: Limb-girdle muscular dystrophy, type 2; MDC1B: Congenital muscular dystrophy 1B; MPRM1: Autosomal dominant myopathy with proximal muscle weakness and early respiratory muscle involvement; HBM: Hyaline body myopathy; MPD3: Adult onset distal myopathy; MFM/ ARVC: Myofibrillar myopathy with arrythmogenic right ventricular cardiomyopathy; NEM6: Nemaline myopathy; MDRV: Distal myopathy with pes cavus and areflexia; MEAX: Myopathy with excessive autophagia.

\section{Competing interest}

The authors report no competing interest. 


\section{Authors' contributions}

GB designed and performed the experiments, analyzed data and wrote the paper. SM performed the experiments and analyzed data. KC, ND and MB designed the experiments and analyzed data. $N D, E G, J B B, A B$ and $L B$ performed the experiments. DS developed analytical tools. IR designed experiments, analyzed data and wrote the paper. All authors read and approved the final manuscript.

\section{Acknowledgements}

This work was supported by the Association Française contre les Myopathies and the Jain Foundation. We thank Dr. Anne Friedrich for technical assistance on gene coordinates and cytogenetic bands studies and Roseline Yao and Julie Dumonceaux from the Inserm U790 for the R9 cells. We are grateful to the sequencing and histological departments of Genethon and to Jean Pascal Lepetit-Stoffaes, Drs Alain Meil, Vincent Collura and Etienne Formstecher from Hybrigenics for excellent assistance.

\section{Author details}

'Généthon CNRS UMR8587, 1, rue de I'Internationale, Evry 91000, France. ${ }^{2}$ Present address: Aix-Marseille Univ and Inserm, UMR 910, Faculté de Médecine Timone, Marseille 13385, France.

Received: 3 August 2012 Accepted: 7 February 2013

Published: 15 February 2013

\section{References}

1. Stelzl U, Worm U, Lalowski M, Haenig C, Brembeck FH, Goehler H, Stroedicke M, Zenkner M, Schoenherr A, Koeppen S, et al: A human protein-protein interaction network: a resource for annotating the proteome. Cell 2005, 122:957-968.

2. Rual JF, Venkatesan K, Hao T, Hirozane-Kishikawa T, Dricot A, Li N, Berriz GF, Gibbons FD, Dreze M, Ayivi-Guedehoussou N, et al: Towards a proteomescale map of the human protein-protein interaction network. Nature 2005, 437:1173-1178.

3. Ewing RM, Chu P, Elisma F, Li H, Taylor P, Climie S, McBroom-Cerajewski L, Robinson MD, O'Connor L, Li M, et al: Large-scale mapping of human protein-protein interactions by mass spectrometry. Mol Syst Biol 2007, 3:89.

4. Bouwmeester T, Bauch A, Ruffner H, Angrand PO, Bergamini G, Croughton K, Cruciat C, Eberhard D, Gagneur J, Ghidelli S, et al: A physical and functional map of the human TNF-alpha/NF-kappa B signal transduction pathway. Nat Cell Biol 2004, 6:97-105.

5. Goehler H, Lalowski M, Stelzl U, Waelter S, Stroedicke M, Worm U, Droege A, Lindenberg KS, Knoblich M, Haenig C, et al: A protein interaction network links GIT1, an enhancer of huntingtin aggregation, to Huntington's disease. Mol Cell 2004, 15:853-865.

6. Lim J, Hao T, Shaw C, Patel AJ, Szabo G, Rual JF, Fisk CJ, Li N, Smolyar A, Hill DE, et al: A protein-protein interaction network for human inherited ataxias and disorders of Purkinje cell degeneration. Cell 2006, 125:801-814.

7. Nigro V, Aurino S, Piluso G: Limb girdle muscular dystrophies: update on genetic diagnosis and therapeutic approaches. Curr Opin Neurol 2011, 24:429-436.

8. Aurino S, Piluso G, Saccone V, Cacciottolo M, D'Amico F, Dionisi M, Totaro A, Belsito A, Di Vicino U, Nigro V: Candidate-gene testing for orphan limbgirdle muscular dystrophies. Acta Myol 2008, 27:90-97.

9. Guglieri M, Straub V, Bushby K, Lochmuller H: Limb-girdle muscular dystrophies. Curr Opin Neurol 2008, 21:576-584.

10. Krogh A, Larsson B, von Heijne G, Sonnhammer EL: Predicting transmembrane protein topology with a hidden Markov model: application to complete genomes. J Mol Biol 2001, 305:567-580.

11. Nielsen H, Engelbrecht J, Brunak S, von Heijne G: A neural network method for identification of prokaryotic and eukaryotic signal peptides and prediction of their cleavage sites. Int J Neural Syst 1997, 8:581-599.

12. Finn RD, Tate J, Mistry J, Coggill PC, Sammut SJ, Hotz HR, Ceric G, Forslund K, Eddy SR, Sonnhammer EL, Bateman A: The Pfam protein families database. Nucleic Acids Res 2008, 36:D281-D288.

13. Vojtek AB, Hollenberg SM: Ras-Raf interaction: two-hybrid analysis. Methods Enzymol 1995, 255:331-342.

14. Formstecher E, Aresta S, Collura V, Hamburger A, Meil A, Trehin A, Reverdy C, Betin V, Maire S, Brun C, et al: Protein interaction mapping: a Drosophila case study. Genome Res 2005, 15:376-384.
15. Fromont-Racine $M$, Rain JC, Legrain P: Toward a functional analysis of the yeast genome through exhaustive two-hybrid screens. Nat Genet 1997, 16:277-282

16. Maiweilidan Y, Klauza I, Kordeli E: Novel interactions of ankyrins-G at the costameres: the muscle-specific Obscurin/Titin-Binding-related Domain (OTBD) binds plectin and filamin C. Exp Cell Res 2011, 317:724-736.

17. Alhamidi M, Kjeldsen Buvang E, Fagerheim T, Brox V, Lindal S, Van Ghelue $\mathrm{M}$, Nilssen O: Fukutin-related protein resides in the Golgi cisternae of skeletal muscle fibres and forms disulfide-linked homodimers via an $\mathrm{N}$ terminal interaction. PLoS One 2011, 6:e22968.

18. Burgo A, Proux-Gillardeaux V, Sotirakis E, Bun P, Casano A, Verraes A, Liem RK, Formstecher E, Coppey-Moisan M, Galli T: A molecular network for the transport of the TI-VAMP/VAMP7 vesicles from cell center to periphery. Dev Cell 2012, 23:166-180.

19. Bolte S, Cordelieres FP: A guided tour into subcellular colocalization analysis in light microscopy. J Microsc 2006, 224:213-232.

20. Quevillon E, Silventoinen V, Pillai S, Harte N, Mulder N, Apweiler R, Lopez R: InterProScan: protein domains identifier. Nucleic Acids Res 2005, 33:W116-W120.

21. Mulder NJ, Apweiler R, Attwood TK, Bairoch A, Bateman A, Binns D, Bork P, Buillard V, Cerutti L, Copley R, et al: New developments in the InterPro database. Nucleic Acids Res 2007, 35:D224-D228.

22. Turner B, Razick S, Turinsky AL, Vlasblom J, Crowdy EK, Cho E, Morrison K Donaldson IM, Wodak SJ: iRefWeb: interactive analysis of consolidated protein interaction data and their supporting evidence. Database (Oxford) 2010, 2010:baq023.

23. Sherman BT, Huang da W, Tan Q, Guo Y, Bour S, Liu D, Stephens R, Baseler MW, Lane HC, Lempicki RA: DAVID Knowledgebase: a gene-centered database integrating heterogeneous gene annotation resources to facilitate high-throughput gene functional analysis. BMC Bioinformatics 2007, 8:426.

24. da Huang W, Sherman BT, Lempicki RA: Systematic and integrative analysis of large gene lists using DAVID bioinformatics resources. Nat Protoc 2009, 4:44-57.

25. Muller A, MacCallum RM, Sternberg MJ: Structural characterization of the human proteome. Genome Res 2002, 12:1625-1641.

26. Hilfiker S, Greengard P, Augustine GJ: Coupling calcium to SNAREmediated synaptic vesicle fusion. Nat Neurosci 1999, 2:104-106.

27. Bansal D, Miyake K, Vogel SS, Groh S, Chen CC, Williamson R, McNeil PL, Campbell KP: Defective membrane repair in dysferlin-deficient muscular dystrophy. Nature 2003, 423:168-172.

28. Vignal E, Blangy A, Martin M, Gauthier-Rouviere C, Fort P: Kinectin is a key effector of RhoG microtubule-dependent cellular activity. Mol Cell Biol 2001, 21:8022-8034

29. Scita G, Nordstrom J, Carbone R, Tenca P, Giardina G, Gutkind S, Bjarnegard M, Betsholtz C, Di Fiore PP: EPS8 and E3B1 transduce signals from Ras to Rac. Nature 1999, 401:290-293.

30. Sarparanta J, Jonson PH, Golzio C, Sandell S, Luque H, Screen M, McDonald K, Stajich JM, Mahjneh I, Vihola A, et al: Mutations affecting the cytoplasmic functions of the co-chaperone DNAJB6 cause limb-girdle muscular dystrophy. Nat Genet 2012, 44:450-455-S451-452.

31. Shannon P, Markiel A, Ozier O, Baliga NS, Wang JT, Ramage D, Amin N, Schwikowski B, Ideker T: Cytoscape: a software environment for integrated models of biomolecular interaction networks. Genome Res 2003, 13:2498-2504.

32. Legrain P, Selig L: Genome-wide protein interaction maps using twohybrid systems. FEBS Lett 2000, 480:32-36.

33. Kahle JJ, Gulbahce N, Shaw CA, Lim J, Hill DE, Barabasi AL, Zoghbi HY: Comparison of an expanded ataxia interactome with patient medical records reveals a relationship between macular degeneration and ataxia. Hum Mol Genet 2011, 20:510-527.

34. Tee JM, Peppelenbosch MP: Anchoring skeletal muscle development and disease: the role of ankyrin repeat domain containing proteins in muscle physiology. Crit Rev Biochem Mol Biol 2010, 45:318-330.

35. Ogut O, Hossain MM, Jin JP: Interactions between nebulin-like motifs and thin filament regulatory proteins. J Biol Chem 2003, 278:3089-3097.

36. Bader GD, Hogue CW: Analyzing yeast protein-protein interaction data obtained from different sources. Nat Biotechnol 2002, 20:991-997.

37. de Morree A, Hensbergen PJ, van Haagen HH, Dragan I, Deelder AM, Hoen PA t, Frants RR, van der Maarel SM: Proteomic analysis of the dysferlin protein complex unveils its importance for sarcolemmal maintenance and integrity. PLoS One 2010, 5:e13854. 
38. van Haagen $\mathrm{HH}$, Hoen PA t, de Morree A, van Roon-Mom WM, Peters DJ, Roos M, Mons B, van Ommen GJ, Schuemie MJ: In silico discovery and experimental validation of new protein-protein interactions. Proteomics 2011, 11:843-853.

39. Cacciottolo M, Belcastro V, Laval S, Bushby K, di Bernardo D, Nigro V: Reverse engineering gene network identifies new dysferlin-interacting proteins. J Biol Chem 2011, 286:5404-5413.

40. Comi GP, Fortunato F, Lucchiari S, Bordoni A, Prelle A, Jann S, Keller A, Ciscato P, Galbiati S, Chiveri L, et al: Beta-enolase deficiency, a new metabolic myopathy of distal glycolysis. Ann Neurol 2001, 50:202-207.

41. Palenzuela L, Andreu AL, Gamez J, Vila MR, Kunimatsu T, Meseguer A, Cervera C, Fernandez Cadenas I, van der Ven PF, Nygaard TG, et al: A novel autosomal dominant limb-girdle muscular dystrophy (LGMD 1F) maps to 7q32.1-32.2. Neurology 2003, 61:404-406.

42. Tang BL, Zhang T, Low DY, Wong ET, Horstmann H, Hong W: Mammalian homologues of yeast sec31p. An ubiquitously expressed form is localized to endoplasmic reticulum (ER) exit sites and is essential for ERGolgi transport. J Biol Chem 2000, 275:13597-13604.

43. Starling A, Kok F, Passos-Bueno MR, Vainzof M, Zatz M: A new form of autosomal dominant limb-girdle muscular dystrophy (LGMD1G) with progressive fingers and toes flexion limitation maps to chromosome 4p21. Eur J Hum Genet 2004, 12:1033-1040.

44. Townley AK, Feng Y, Schmidt K, Carter DA, Porter R, Verkade P, Stephens DJ: Efficient coupling of Sec23-Sec24 to Sec13-Sec31 drives COPIIdependent collagen secretion and is essential for normal craniofacial development. J Cell Sci 2008, 121:3025-3034.

45. Kramerova I, Kudryashova E, Venkatraman G, Spencer MJ: Calpain 3 participates in sarcomere remodeling by acting upstream of the ubiquitin-proteasome pathway. Hum Mol Genet 2007, 16:1006.

46. Hack AA, Cordier L, Shoturma DI, Lam MY, Sweeney HL, McNally EM: Muscle degeneration without mechanical injury in sarcoglycan deficiency. Proc Natl Acad Sci U S A 1999, 96:10723-10728.

47. Barton ER: Restoration of gamma-sarcoglycan localization and mechanical signal transduction are independent in murine skeletal muscle. J Biol Chem 2010, 285:17263-17270.

48. Knoll R, Linke WA, Zou P, Miocic S, Kostin S, Buyandelger B, Ku CH, Neef S, Bug M, Schafer $\mathrm{K}$, et al: Telethonin deficiency is associated with maladaptation to biomechanical stress in the mammalian heart. Circ Res 2011, 109:758-769.

doi:10.1186/2044-5040-3-3

Cite this article as: Blandin et al:: A human skeletal muscle interactome centered on proteins involved in muscular dystrophies: LGMD interactome. Skeletal Muscle 2013 3:3.

\section{Submit your next manuscript to BioMed Central and take full advantage of:}

- Convenient online submission

- Thorough peer review

- No space constraints or color figure charges

- Immediate publication on acceptance

- Inclusion in PubMed, CAS, Scopus and Google Scholar

- Research which is freely available for redistribution 